\title{
RECENT ADVANCES IN THE SYNTHESIS OF SPECIALIZED HETEROCYCLIC MOLECULES BY 1, 3-DIPOLAR CYCLOADDITION REACTION: A STUDY BASED ON GREENER PROTOCOLS
}

\author{
Bhaskar Chakraborty ${ }^{1,2}$ \\ ${ }^{1}$ Organic Chemistry Laboratory, Sikkim Government College (NBBDC), \\ Gangtok 737102, Sikkim, India \\ ${ }^{2}$ Academic Visitor, School of Chemistry, Cardiff University, Park Place, \\ Cardiff CF10 3AT, Wales, UK \\ E-mail: bhaskargtk@yahoo.com
}

\begin{abstract}
In today's scenario trying newer methods or concepts in organic synthesis leading to the generation of new heterocycles and also saving the environment is equally challenging indeed for an organic chemist. Keeping in mind we feel that "green chemistry" methodologies are the need of the hour to save our environment and mankind. The aim of this review is to provide an overview of green chemistry protocols for the synthesis of a new class of specialized heterocyclic molecules via 1,3-dipolar cycloaddition reaction. In the present review, particular attention has been focused on the application of mechanochemical procedures and the use of green solvents. In addition, the review also includes reactions performed in ionic liquids, microwave-induced reactions, aqueous phase synthesis and light-induced reactions reported recently. This account also reports about the recent green synthetic methodologies of new nitrones and their cycloaddition reactions performed in our and other laboratories. We have also explored several protocols of atom efficient reactions of nitrones and further application of cycloadducts using green chemistry methodologies. The advantages of these procedures over existing conventional methodologies and the effect of solvents have been focused in this review to highlight different aspects of environmental issues and also in the selectivity of the reactions.

Keywords: Green Chemistry, 1,3-Dipolar cycloadditions, Heterocycles, Mechanochemistry, Aqueous Phase Synthesis, Ionic Liquids, Microwave induced Reactions
\end{abstract}

(C) RASĀYAN. All rights reserved

\section{INTRODUCTION}

Synthesis of a new heterocyclic molecule with potential biological activity is one of the most attractive areas of research nowadays. The most widely used and popular reaction for this purpose is 1,3-dipolar cycloaddition which represents the unique method for the construction of five-membered heterocycles, commonly called isoxazolidine \& isoxazoline derivatives. Majority of them are the important frameworks of various natural products with potential medicinal activities like antibacterial, anticonvulsant, antibiotic, antitubercular and anticancer respectively. ${ }^{1-3}$ Unfortunately, the majority of 1,3-dipolar cycloaddition reactions require drastic reaction conditions in conventional methodologies due to the use of many health-hazardous solvents and catalysts. Therefore, not only the chemists engaged in these reactions are affected but simultaneously our environment is found to have an impact as well. Hence conducting reaction for the synthesis of a new heterocycle and at the same time saving our environment is equally important.

Before discussing different greener methodologies for the synthesis of heterocyclic molecules, we should have a brief overview of the different theories (molecular orbital theory, frontier molecular orbital theory 
RASĀYAN J. Chem.

Vol. 12 | No. 4 |2183 - 2209| October - December | 2019

related to regio and stereoselectivity) of 1,3-dipolar cycloaddition reaction and also the general methods of synthesis of nitrone and cycloadducts (isoxazolidine and isoxazoline derivatives).

On the basis of mechanistic investigations, K. N. Houk etal ${ }^{4}$ pointed out that cycloaddition of 1,3-dipole to dipolarophile (alkene) is stereospecifically syn and solvent polarity has little effect on the reaction rate as well as small activation of enthalpy. This theory agrees with concerted five centered mechanisms and found compatible with reactivity and regioselectivity. Based on orbital symmetry consideration, theoretical evidence has been provided for the concerted mechanism in connection with cycloaddition of [ $4 \pi$ 's and $6 \pi$ 's] but not for the [ $4 \pi$ 's $+4 \pi$ 's] cycloaddition of 1,3-dipole to diene. But it has been found that experimentally observed regioselectivity of most of the 1,3-dipolar cycloaddition reactions is the most difficult phenomenon to explain.

Using frontier molecular orbital theory (FMO approach) and also the generalized frontier orbitals of 1,3dipoles and dipolarophiles, Houk etal ${ }^{4}$ solved the problem of regioselectivity. Sustman ${ }^{5}$ suggested that depending upon the symmetry properties of the HOMO and LUMO orbitals of the dienes and dipolarophiles we may judge if the 1,3-dipolar cycloaddition reaction is allowed or forbidden.

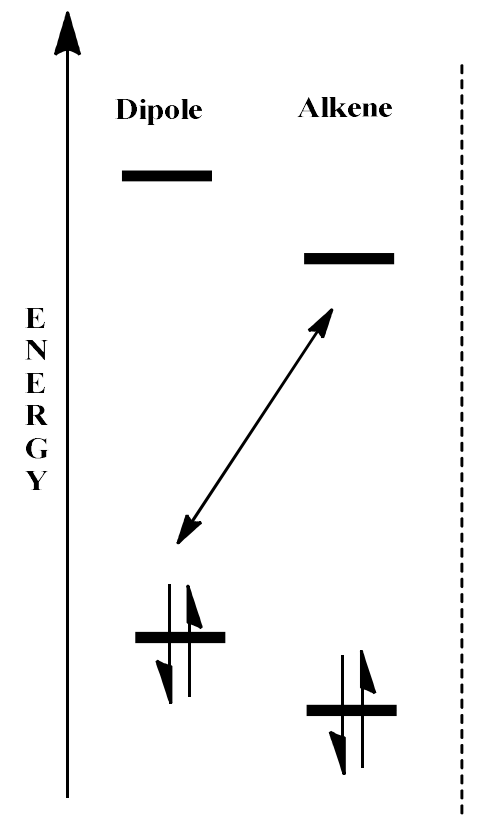

Type I

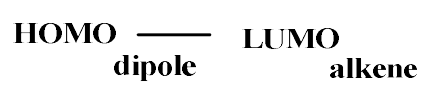

Types

of

dipoles

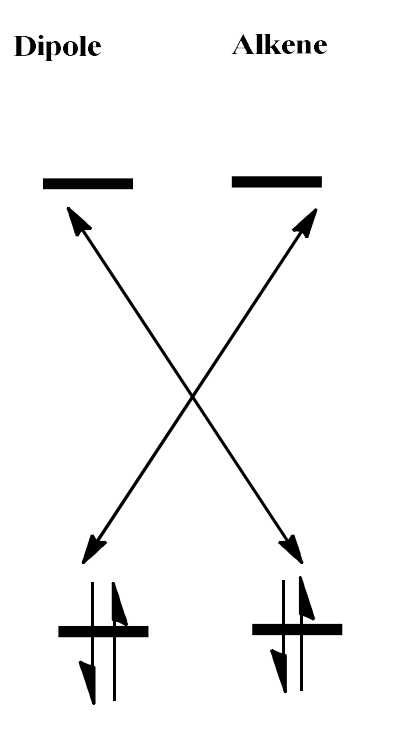

Type II

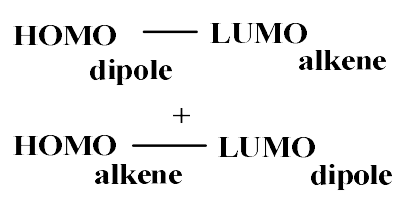

nitrones, nitrile oxides
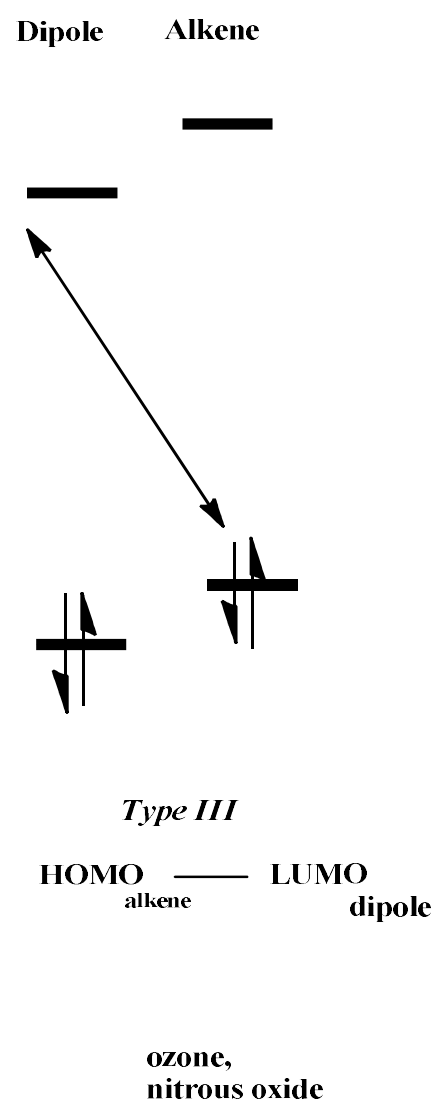

Scheme-1: FMO Approach of HOMO-LUMO Interactions

- Type I: Represents dominant interaction between HOMO (dipole)-LUMO (dipolarophiles).

- Type II: Represents LUMO (dipole)-HOMO (dipolarophiles). But in type II, both the LUMO (dipole)-HOMO (dipolarophiles) and HOMO (dipole)-LUMO (dipolarophiles) are important in determining reactivity and regioselectivity. Type I dipoles are those having high lying HOMO's and LUMO's and referred to as HOMO controlled or nucleophilic 1,3-dipoles. It is referred to as HUMOLUMO controlled dipoles.

- Type III: Represents low lying FMO's and referred to as LUMO controlled or electrophilic dipoles. 
RASĀYAN J. Chem.

Vol. 12 | No. 4 |2183 - 2209| October - December | 2019

Molecular Orbital Theory Behind 1,3-Dipolar Cycloaddition Reactions

Lewis Acid Activation

Coordination of LA to either the Dipole or the Alkene Results in LUMO Lowering and A Faster Reaction Rate

K.N. Houk ${ }^{4}$ has successfully applied this simple model of FMO approach to all common 1,3-dipoles and shown that the theory satisfactorily explains all the experimental results. The HOMO controlled 1,3 dipoles (nitrile ylides, diazoalkanes and azomethine ylides) are found to react readily with alkenes having one or more electron-withdrawing substituents. On the other, the nitrile imines, azides, and azomethyne imines are HOMO-LUMO controlled dipoles that react rapidly with both electron-rich and electrondeficient dipolarophiles. The nitrone and nitrile oxides are also HOMO-LUMO controlled dipoles but these species are distorted towards the LUMO controlled side. The species nitrous oxide and ozone with several electronegative atoms are LUMO controlled 1,3 dipoles. Generally, depending upon the nature of the dipole and dipolarophile, the 1,3-dipolar cycloaddition reaction is controlled either by a LUMO (dipolarophile)-HOMO (dipole) or a LUMO (dipole)-HOMO (dipolarophile) interaction. But in some cases, a combination of both interactions are involved. The interactions of the dipole (LUMO) with a dipolarophile (HOMO) favor the formation of the cycloadducts with the substituent on carbon atom adjacent to $z$ while the opposite frontier orbital interaction favors opposite regioisomers. The HOMO's of the 1,3 dipoles are generally found to have larger terminal co-efficient on the group $z$ while the LUMO's have larger co-efficient at the opposite terminus. The HOMO's and LUMO's of 1,3 dipole are similar to those of allyl anion. When the two terminals differ greatly in electronegativity, more differences in terminal co-efficient takes place.

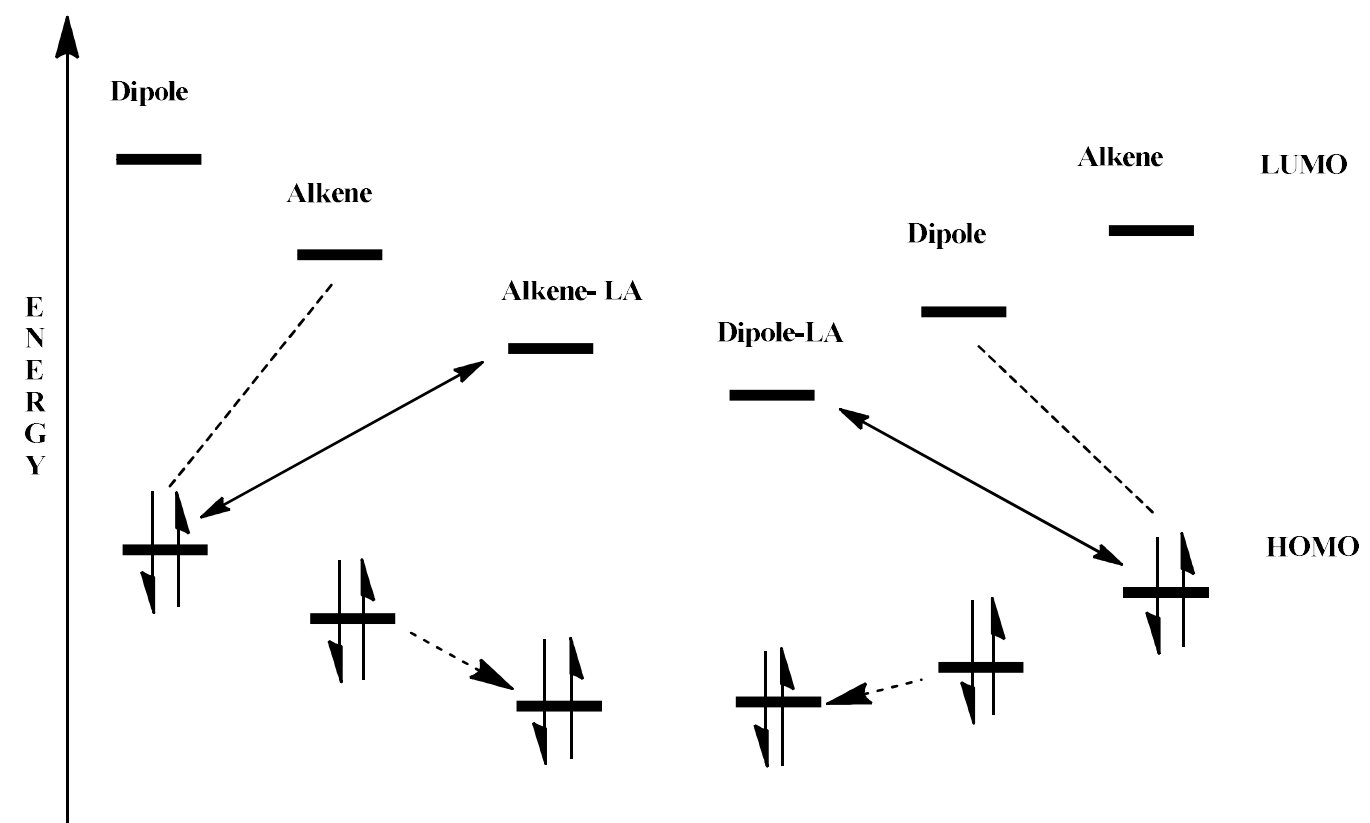

Scheme-2: HOMO-LUMO of Dipole and Dipolarophiles

Nitrones and nitrile oxides react to give mainly the five substituted cycloadducts with weakly electrondeficient alkenes like acrylonitrile and ethyl acrylate. The HOMO's and LUMO's of these electrondeficient alkenes interact fairly with the LUMO's and HOMO's of the nitrones and nitrile oxides. Their orientation is also influenced by both interactions. From the experimental results, it has been found that the dipole LUMO - dipolarophile HOMO has more influence on regioselectivity. Huisgen ${ }^{6}$ observed that acetylenic dipolarophiles are less reactive than expected due to their ionization potentials. Compared to alkenes, alkynes have large HOMO - LUMO gap therefore, it is expected that during interactions with the alkyne, LUMO plays the most significant part and hence alkynes are less reactive than expected. However, the reactivity of various nitrones with electron-deficient alkynes and alkenes are actually determined by 
RASĀYAN J. Chem.

Vol. 12 | No. 4 |2183 - 2209| October - December | 2019

dipole (HOMO)-dipolarophile (LUMO) interactions and also the regiochemistry. In case of alkyne, the system is controlled by dipole (LUMO) and the dipole (HOMO)-dipolarophile (LUMO) interactions become so important that it dominates the reaction and leads to the formation of 4-substituted adducts only. If both the dipole and dipolarophile is nonsymmetrical in nature, regioisomeric adducts may be formed.

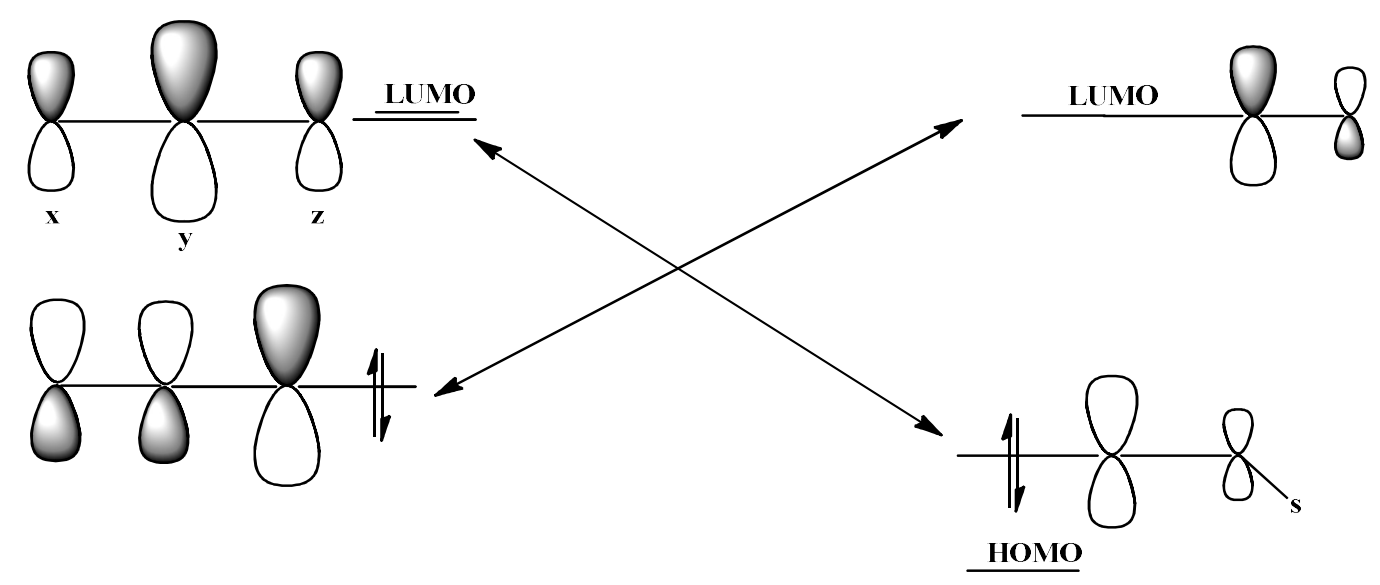

Scheme-3: HOMO and LUMO'S of 1,3 Dipole

\section{Stereoselectivity In Nitrone Cycloaddition Reactions}

Generally, the additions of nitrones to dipolarophiles are cis in nature, so the relative stereochemistry at C4 and $C-5$ protons are always determined by the geometrical relationship of the substituents on the alkene moiety. The syn and anti isomers of dipole (stability and proportion of each depend upon steric considerations and hydrogen bonding etc) may lead to diastereomeric products depending on the approach of dipole and dipolarophile. Exo / endo approach of the dipolarophiles is also very important and needs to be considered. Secondary orbital interactions are not as relevant as needed in Diels-Alder reactions but steric interactions are important. Therefore, the exo product is a more stable product.

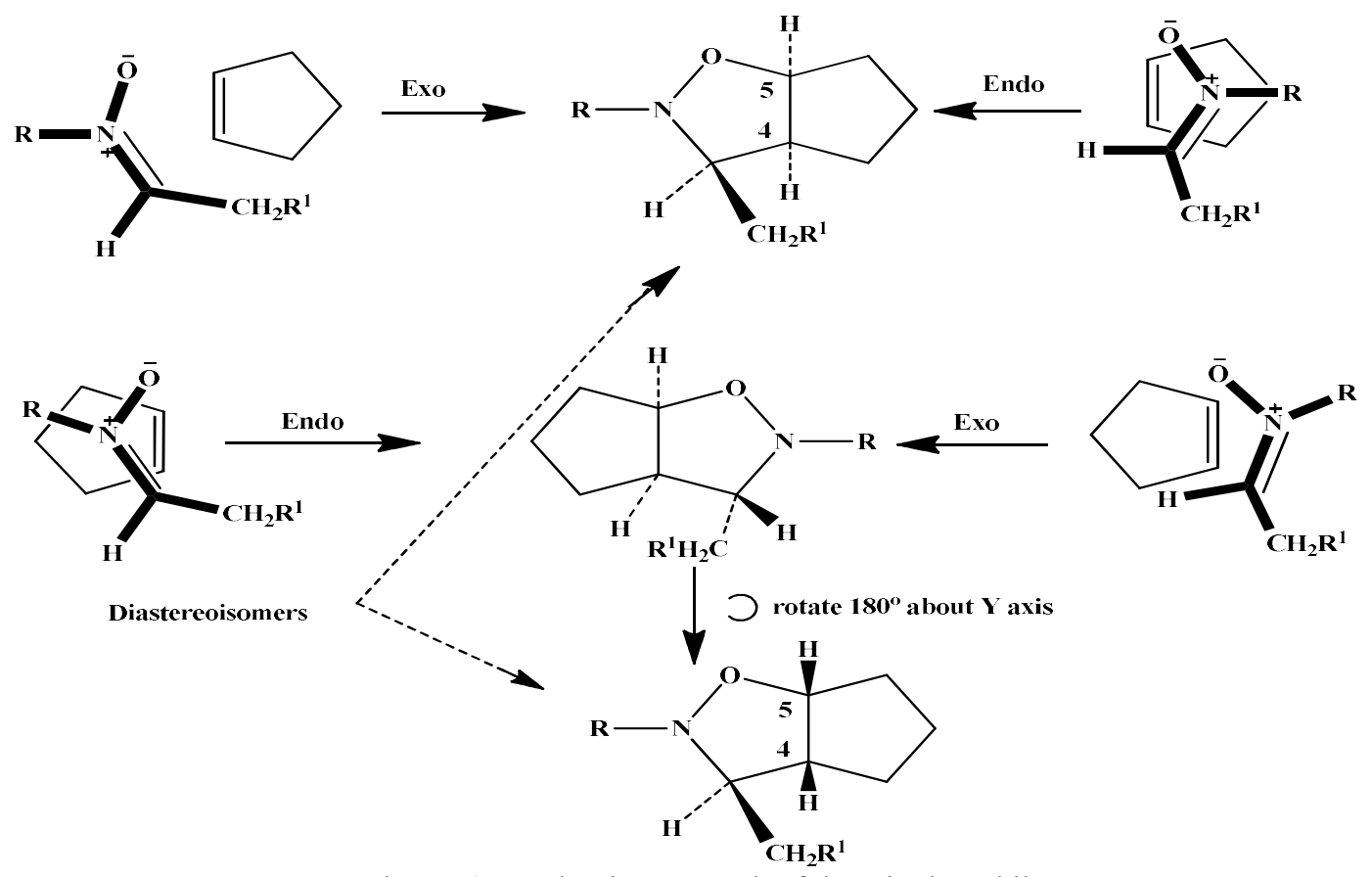

Scheme-4: Exo/Endo Approach of the Dipolarophiles 
RASĀYAN J. Chem.

Vol. 12 | No. 4 |2183 - 2209| October - December | 2019

In nitrone-alkene cycloaddition reaction, two pairs of regioisomeric and diastereomeric products may develop. This is due to the approach of both the nitrone and alkene to each other in two regiochemical aspects viz, an endo or exofashion ${ }^{7}$ (Scheme-4).

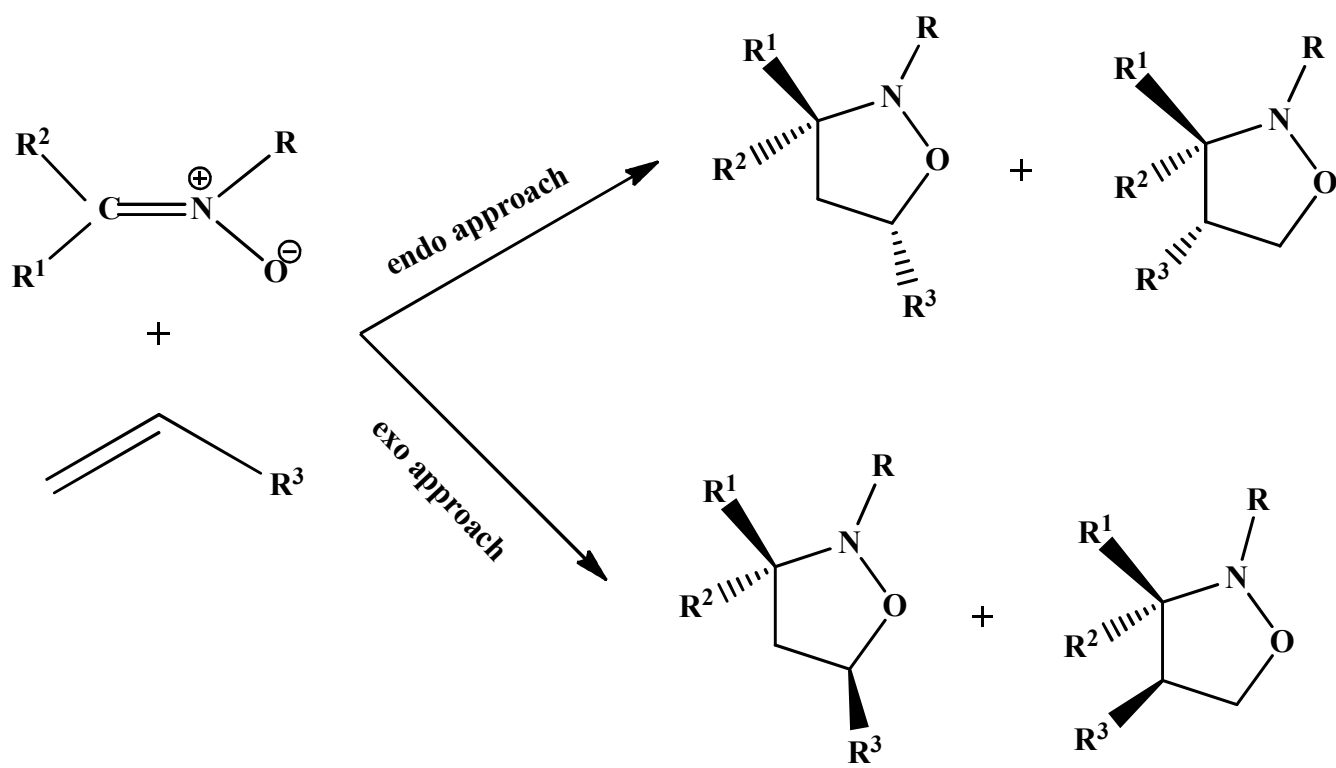

Scheme-5: Exo/Endo Approach of Nitrone and Alkene

Therefore, both regioselectivity and stereoselectivity are highly relevant in inter and intramolecular cycloaddition reactions of nitrone and alkenes. The endo and exo terminology is well known from the Diels-Alder reaction. ${ }^{8}$ The endo isomer results from the reaction in which the nitrogen atom of the dipole points in the same direction as the substituent of the alkene. However, the endo transition state in the Diels-Alder reaction is stabilized by secondary $\pi$-orbital interaction. The actual interaction is between the nitrone $\mathrm{p}_{\mathrm{z}}$ orbital with a vicinal $\mathrm{p}_{\mathrm{z}}$ orbital on the alkene and thus the stabilization is small. ${ }^{9}$ The endo/exo selectivity in the 1,3-dipolar cycloaddition reaction is primarily controlled by the structure of the substrates or by the presence of catalyst. It has to be noted that endo/exo assignment of the products is misleading for the reactions in which the nitrone can undergo $Z / E$-interconversion and therefore $c i s$ or trans terminology should be used.

\section{Regioselectivity in Nitrone Cycloaddition Reactions}

Although regioselectivity is controlled by both steric and electronic effects ${ }^{10}$, the steric effect may be overruled by strong electronics effects. ${ }^{11}$ The 5 -substituted adduct of the cycloaddition reaction results when electron-rich or electron-neutral alkenes react with nitrones. The cycloaddition reaction is primarily controlled by the lowest unoccupied molecular orbital (LUMO) dipole and the highest occupied molecular orbital (HOMO) dipolarophile interaction. The nitrone and alkene react in a regioselective manner resulting in 5-substituted isoxazolidine because the LUMO (dipole) has the largest coefficient at the carbon atom and the HOMO alkene has the largest coefficient at the terminal carbon atom. This is supported by steric factors. It has been observed that for the alkenes with an electron-withdrawing Group (EWG), the reaction is primarily controlled by the HOMO (dipole) - LUMO (dipolarophile) interaction. In this case, the HOMO (dipole) has the largest coefficient at the oxygen atom and the LUMO (dipolarophile) has the largest coefficient at the terminal carbon atom. This situation helps in favor of the development of the 4-substituted adducts. But since steric factors oppose this, therefore mixtures of regioisomers are often obtained ${ }^{12}$. However, the steric factor is eliminated in the reaction of nitrones with 1,2-disubstituted alkenes having an electron-withdrawing group. This leads to the development of frontier molecular orbital (FMO) controlled regioselectivity of the reaction with the development of 4-EWG substituted isomer as the sole product (Scheme-6). 
RASĀYAN J. Chem.

Vol. 12 | No. 4 |2183 - 2209| October - December | 2019

\section{1, 3-dipole}

1,3-dipole participates in the [3+2] cycloaddition reaction to develop 5-membered ring systems. The reaction is analogous to the Diels-Alder reaction which forms 6 membered rings. The reactive partners in this cycloaddition reaction are 1,3-dipoles and dipolarophiles (diene and dienophile in the Diels-Alder reaction). This is a $4 \pi+2 \pi$ process as well.
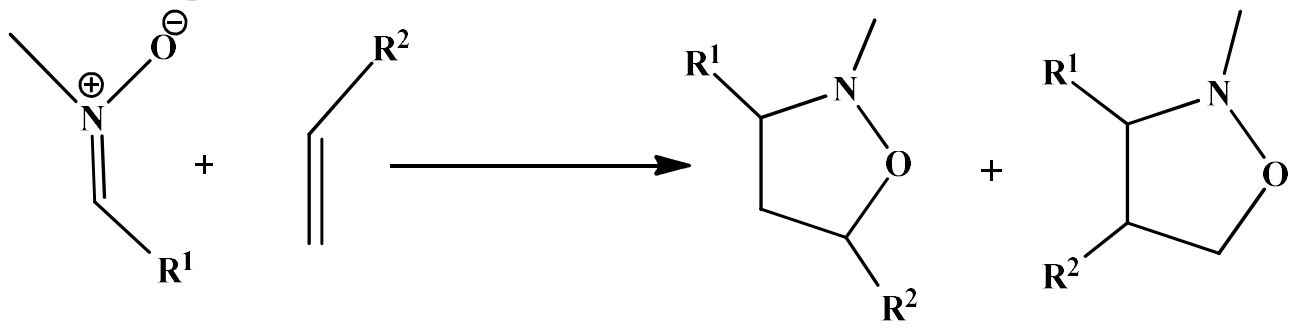

$\mathbf{R}^{2}=$ Electron withdrawing gr (EWG) / Electron donating group (EDG)

Scheme-6: FMO-Controlled Regioselectivity
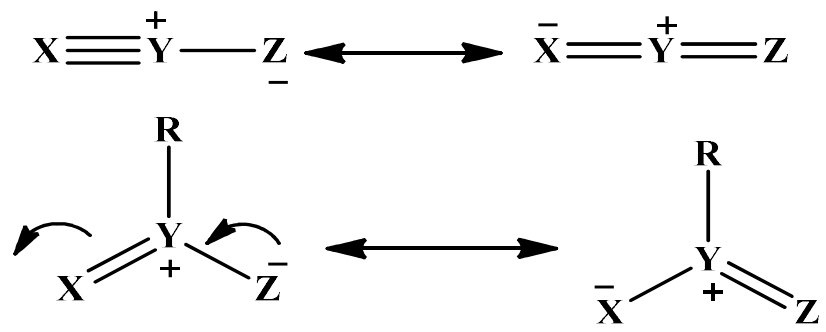

$\mathrm{X}, \mathrm{Y}, \mathrm{Z}=\mathrm{C}, \mathbf{N}, \mathbf{O}, \mathrm{S}, \mathrm{P} \ldots \ldots .$.

Scheme-7: Resonance in Dipole and Dipolarophiles

The stabilities of 1, 3-dipoles vary greatly. Some of them can be isolated and stored while others are relatively stable. Usually, they are prepared the same day for their use in cycloaddition reactions. There are two general classes of dipole and are referred to as $s p^{2}$ and $s p$ hybridized dipoles.

sp-dipole (linear dipoles like the propargyl anion)

Azide

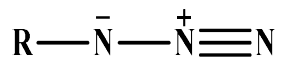<smiles>[R]N=[N+]=[Ni]</smiles>

Nitrile imide (Nitrile imine)

Nitrile oxide
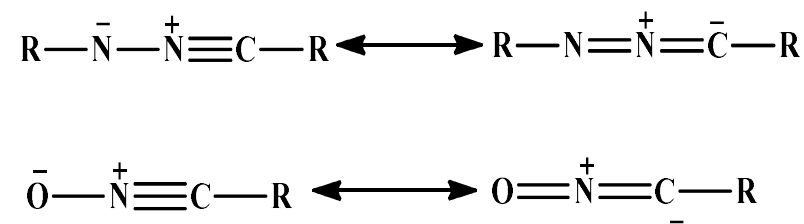<smiles>[R]C=[N+]=O</smiles>

Nitrile ylide<smiles>[R]C#[N+][X]([R])[R]</smiles><smiles>[R]C=[N+]=C([R])[R]</smiles>

Diazoalkane<smiles></smiles><smiles>[R]C([R])=[N+]=[N-]</smiles>

stable if aromatic alkyl azides can explode

generated and used in-situ generated and used in-situ

generated and used insitu

relatively stable. diazomethane can be stored

Scheme-8: Resonance in sp-Dipoles 
RASĀYAN J. Chem.

Vol. 12 | No. 4 |2183 - 2209| October - December | 2019

$\mathbf{s p}^{2}$-dipole (bent dipoles like the allyl anion)

Ozone

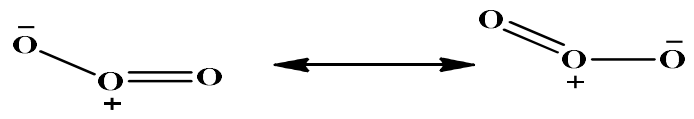

generated \& used in situ

Nitrones<smiles>[R]C([R])=[N+]([R])[C-]CC([R])[R]</smiles>

relatively stable but reactions are done in situ
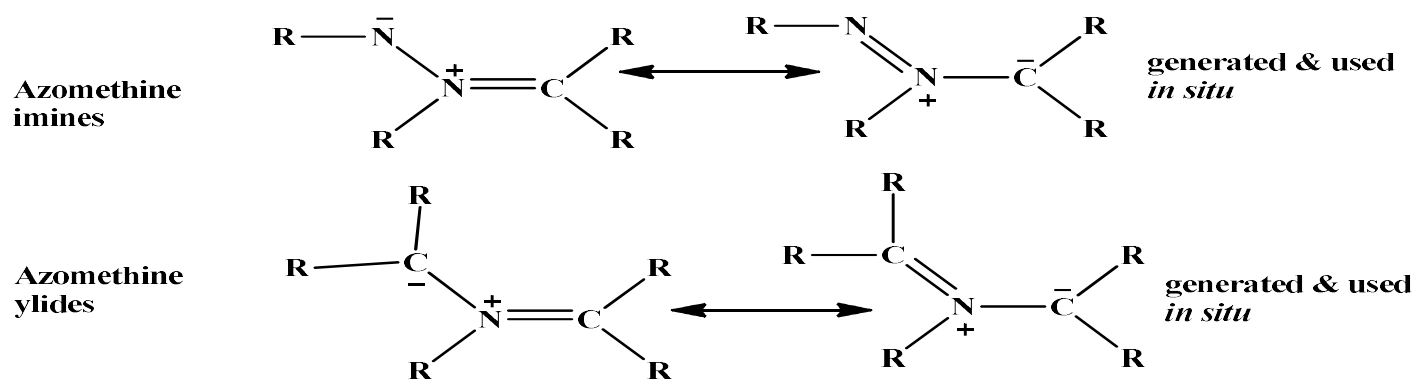

Scheme-9: Resonance in sp2 Dipoles
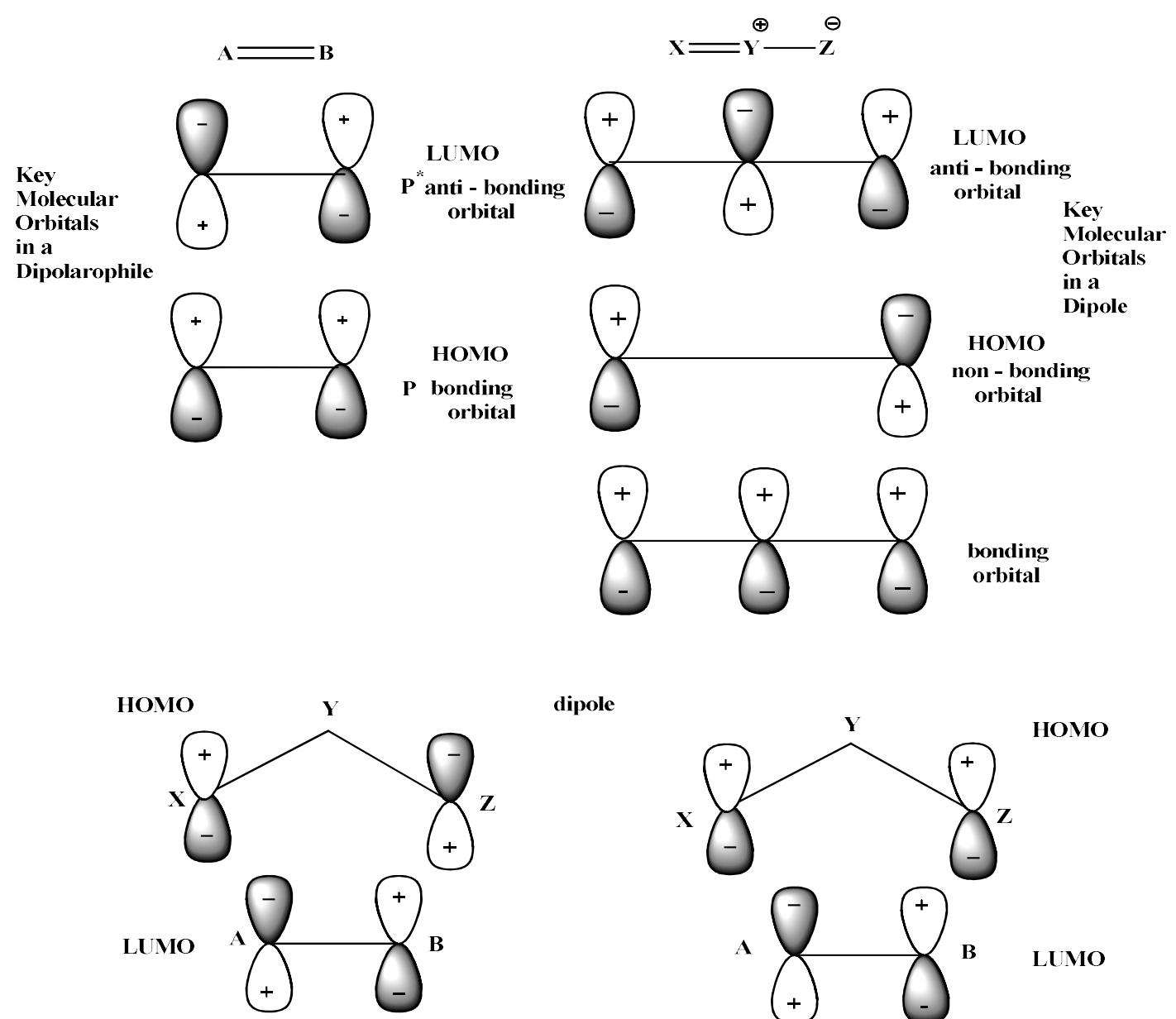

dipole

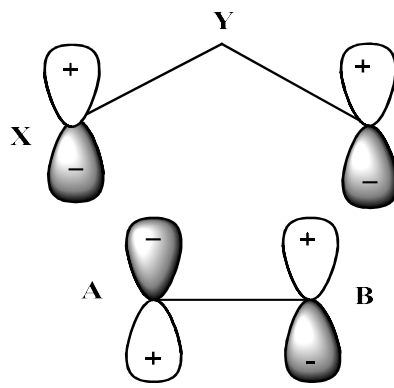

HOMO

Scheme-10: HOMO and LUMO'S of Dipole and Dipolarophiles 
RASĀYAN J. Chem.

Vol. 12 | No. 4 |2183 - 2209| October - December | 2019

\section{Reactivity Profile of 1, 3-Dipoles}

The general profile of the reaction between dipoles and dipolarophiles are represented as follows:

(a) It is accepted that cycloaddition reactions are concerted in nature i.e. they have no distinct intermediates but the bond formation may be asynchronous.

(b) The reaction rates are not influenced by solvent polarity. So there is little change in polarity between reactants and transition state.

The rate of reaction between dipoles and dipolarophiles vary considerably. This can be explained by K. Fukui's Frontier Molecular Orbital Theory (FMO approach) which considers the interaction between molecular orbitals of the dipole and dipolarophiles.

The term "nitrone" was coined by Pfeiffer in 1916 from nitrogen ketone (azomethine oxide) so as to keep its resemblance to the carbonyl group in its several reactions ${ }^{1}$.

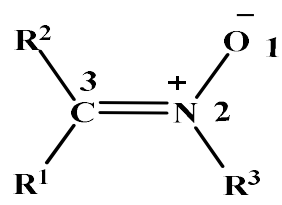

\section{Scheme-11: General Structure of Nitrone}

Nitrones are versatile synthetic intermediates and excellent spin trapping reagents. ${ }^{1}$ This is mainly due to their reactivity as dipoles in 1,3-dipolar cycloaddition reaction which is known for more than 80 years. ${ }^{1}$ The reactivity of nitrones as electrophiles in nucleophilic addition reactions have been also known for more than 60 years. ${ }^{13}$ This reaction has been developed ${ }^{14}$ together with other less conventional reactions ${ }^{15}$ including radical additions ${ }^{16}$ only during the last two decades (Scheme-12).

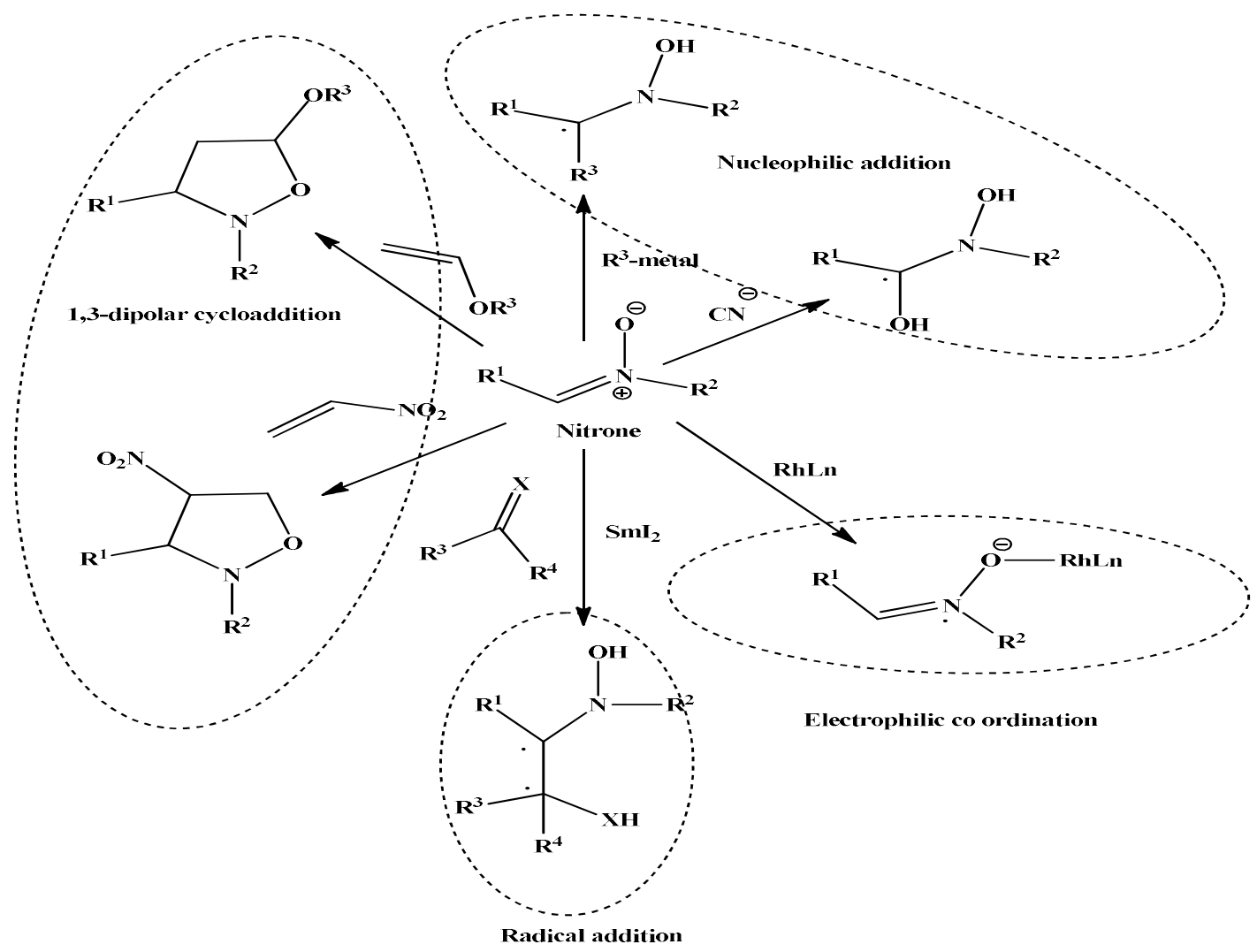

Scheme-12: Various Reactions of Nitrones 
RASĀYAN J. Chem.

Vol. 12 | No. 4 |2183 - 2209| October - December | 2019

A nitrone is a 1,3-dipole in 1, 3-dipolar cycloaddition reaction. It reacts with alkenes to form Isoxazolidines and the scheme may be shown as follows:

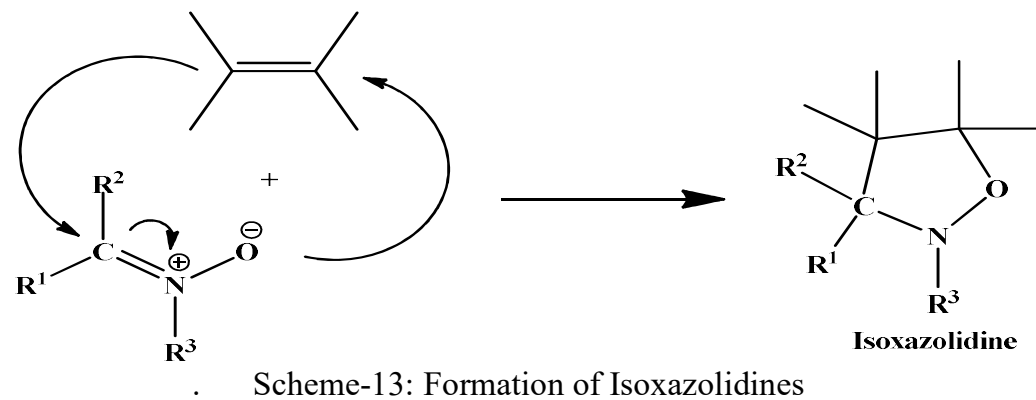

Similarly, the nitrone can react as 1,3-dipole with alkynes in 1,3-dipolar cycloaddition reaction to form Isoxazolines. The scheme may be represented as follows:

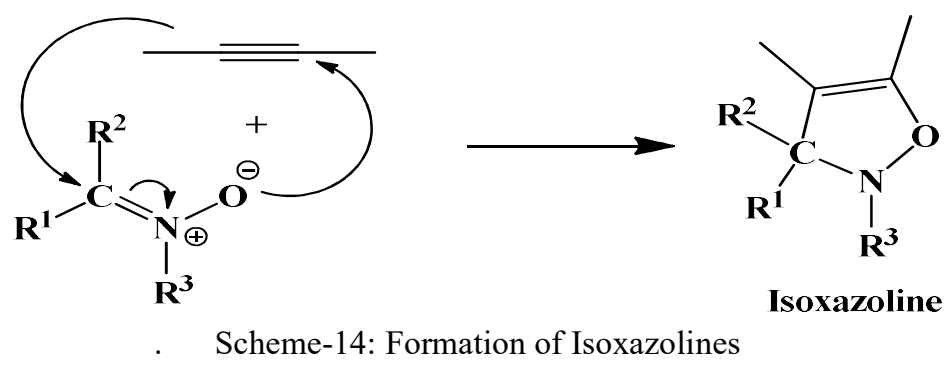

In allyl type of 1,3-dipole, if we restrict the atom a,b,c to carbon, nitrogen, and oxygen, the nitrone results.

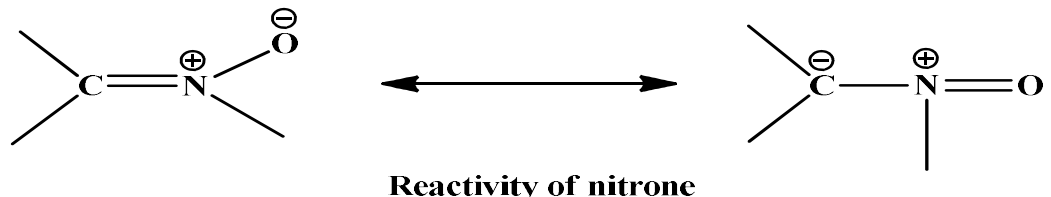

Structural Investigations of Nitrones: X-Ray Crystallographic Studies

Determination of the structure of a nitrone is always a point of interest. Structures of few nitrones have been determined by Folting, Lipscomb and Jerslev using X-ray crystallography. The trans disposition of the aryl and methyl groups and $Z$ configuration of the $\mathrm{C}$-(4-chlorophenyl)- $N$-methyl nitrone ${ }^{17}$ was confirmed by the X-ray crystallography. The N-O bond length in the nitrone has been found to be $1.284 \AA$ which is considerably shorter than the N-O distance in isomeric syn oxime $(1.480 \AA)$ indicating the partial double bond character of this bond in the nitrone. Moreover, the $\mathrm{C}=\mathrm{N}$ distance is $1.309 \AA$ in the nitrone which is longer than the corresponding bond in the O-methyloxime $1.260 \AA$. This indicates a reduction of double bond character (Scheme-15). The corresponding data for $\alpha, \alpha, \mathrm{N}$-triphenyl nitrone are indicated in the formula ${ }^{18}$.

\section{The Concept And Importance of Green Chemistry In Nitrone Cycloaddition Reactions}

The recent reviews on nitrone cycloaddition reactions ${ }^{14 a, 14 b, 15 a}$ (from January 2000 - May 2019) reveals that majority of the reactions are conducted following "Green Chemistry" methodologies. ${ }^{19}$ Environment friendliness and sustainable development is being the need of the hour rather than using conventional solvents like benzene, dichloromethane, tetrahydrofuran, etc. Synthesis of nitrone and their cycloaddition reactions are mainly performed by the following methodologies as under nowadays:

- Mechanochemical synthesis and cycloaddition reactions of nitrones

- Aqueous phase 1,3-dipolar cycloaddition reactions (in water \& on water reactions) 
RASĀYAN J. Chem.

Vol. 12 | No. 4 |2183 - 2209| October - December | 2019

- Microwave-assisted (solid phase) synthesis of nitrones and cycloaddition reactions

- Ionic liquid (RTIL) mediated 1,3-dipolar cycloaddition reactions

- To utilize side products in further reactions (atom efficiency)
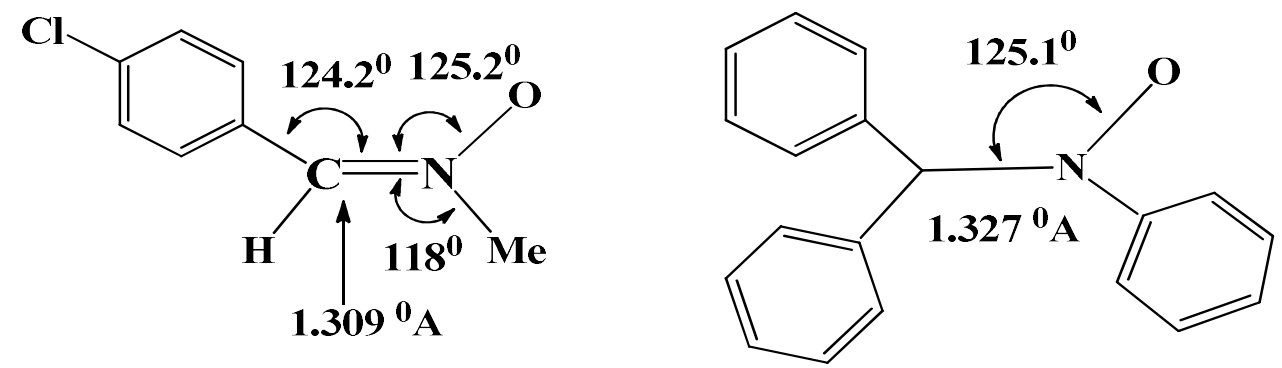

Scheme-15: Structural Data of Two Different Nitrones

In the majority of the reactions, mild reaction conditions (room temperature, short reaction times, avoiding oil bath heating etc), easy workup and isolation of the compounds have made these protocols much more attractive.

The aim of this account is to provide an overview of green chemistry protocols for the synthesis of specialized heterocycles (isoxazolidine \&isoxazoline derivatives) via 1,3-dipolar cycloaddition reactions. We have not included the azides-alkynes cycloadditions which are well-known as Huisgen reactions because of their large presence in literature both in the original publication and in review. In the present account, the primary attention has been focused on the mechanochemical procedure and use of green solvents, solventless reactions. This review also tried to highlight the latest developments in cycloaddition reactions performed with ionic liquids, water and under microwave irradiated reactions. The advantages of these greener protocols over the conventional procedures have been described.

The famous flow-chart of green chemistry recommended by Paul Anastas and W. Warner in their famous book ${ }^{19}$ is very much relevant as a part of this review (Flow Chart-1).

\section{Synthesis of Nitrones}

Nitrones can be easily prepared by oxidation reactions, condensation reactions of $N$-monosubstituted hydroxylamine's, reactions of oximes with electrophiles and other various methods. Along with known methodologies few new approaches are enlisted here which are new and have importance in cycloaddition reactions.

1. Beauchemin et $\mathrm{al}^{20}$ have shown that the synthesis of ketonitrones from monosubstituted allenes may be performed using a Cope-type hydroamination reaction (Scheme.-16).

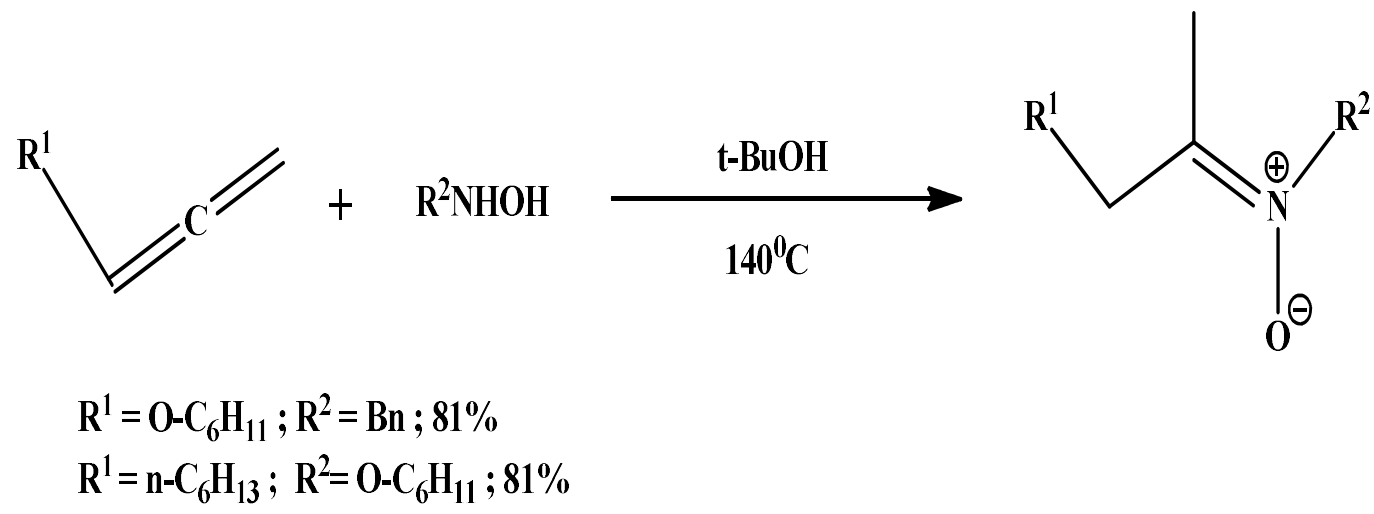

Scheme-16: Synthesis of Keto Nitrone From Allenes 
RASĀYAN J. Chem.

Vol. 12 | No. 4 |2183 - 2209| October - December | 2019

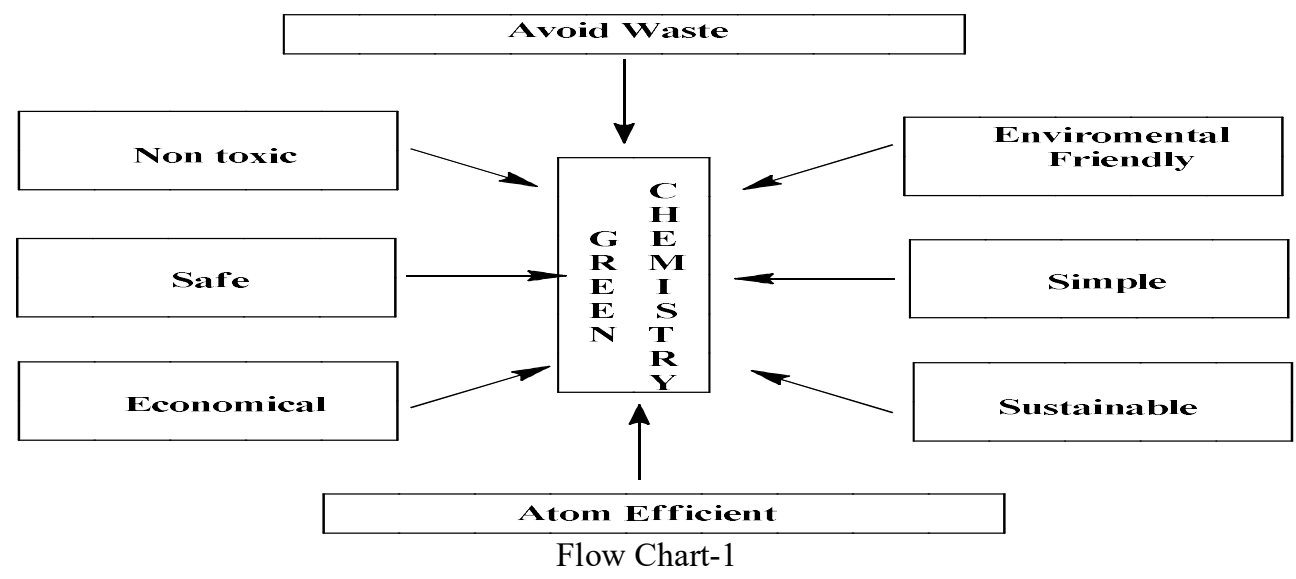

2. Zhang et $\mathrm{al}^{21}$ have reported that the cyclization of $N$-(2-perfluoroalkyl-3-alkynyl) hydroxylamines catalyzed by AgOTf provides 4-perfluoroalkyl substituted 1-pyrroline $N$-oxides (Scheme 17).

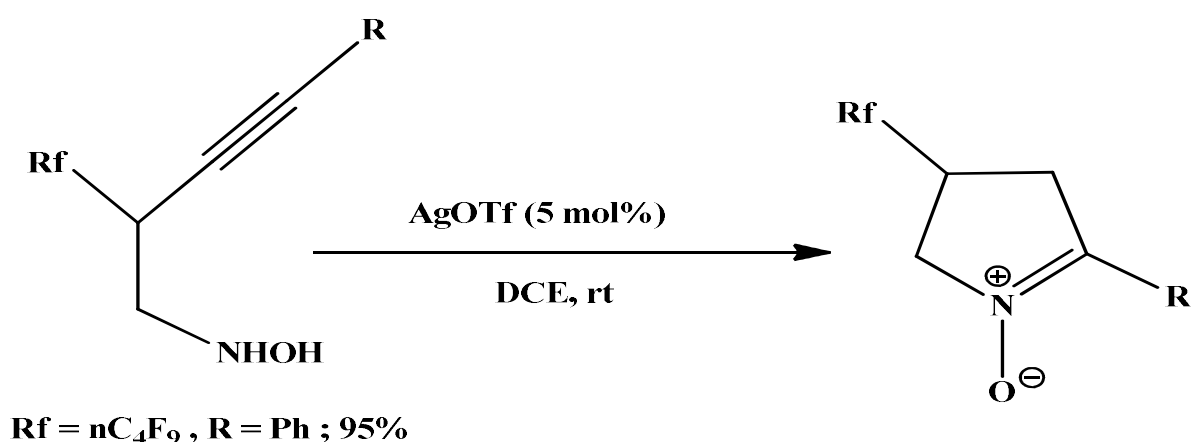

$R f=C F_{3}, R=4-\mathrm{NO}_{2}-C_{6} H_{4} ; 98 \%$

Scheme-17: Synthesis of New N-Oxides From Alkynes

3. A new synthetic route for the synthesis of $N$-alkylated $\alpha, \beta$-unsaturated ketonitrones is recently reported by Terada et al. ${ }^{22}$ They have used propargyloxyamines as the precursor in the presence of $\mathrm{CuI}$ catalysts in the synthesis of nitrone. The reaction can be rationalized by assuming coppercatalyzed intramolecular hydroamination followed by electrocyclic ring-opening of 3-isoxazoline (Scheme-18).

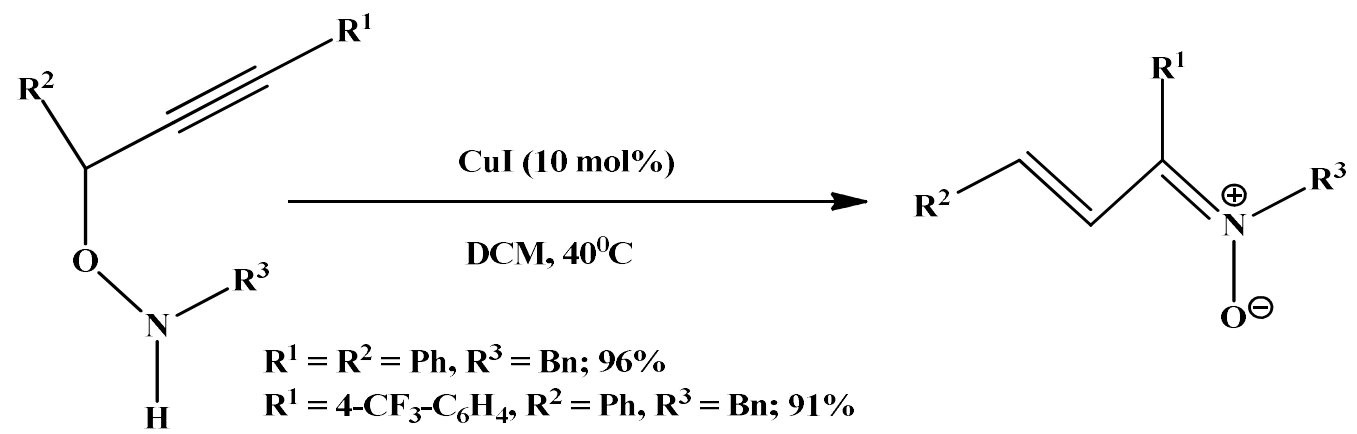

Scheme-18: Synthesis of Unsaturated Nitrones From Alkyne Derivatives

4. Synthesis of cyclic nitrones of interest has been reported by Molins et al. ${ }^{23}$ The reaction is achieved by the oxidation of bicyclic isoxazolidines using m-CPBA with excellent yields 
(Scheme-19).
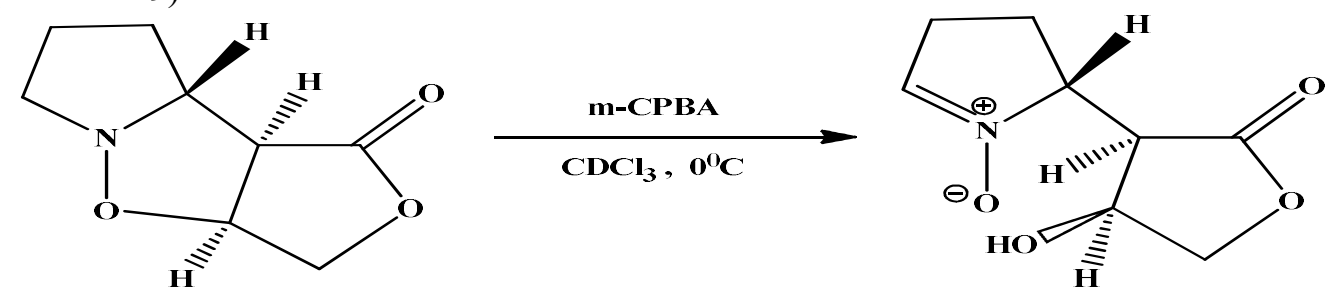

Scheme-19: Synthesis of Cyclic Nitrones From Oxidation of Bicyclic Isoxazolidine

5. Few new nitrones of special interest have been also reported by Chakraborty et.al ${ }^{24}$ following green chemistry methodologies. $\alpha$-amino nitrone is one of them and it has been synthesized successfully from formamide using microwave-induced reaction. This is a new approach to the synthesis of nitrone (Scheme-20).

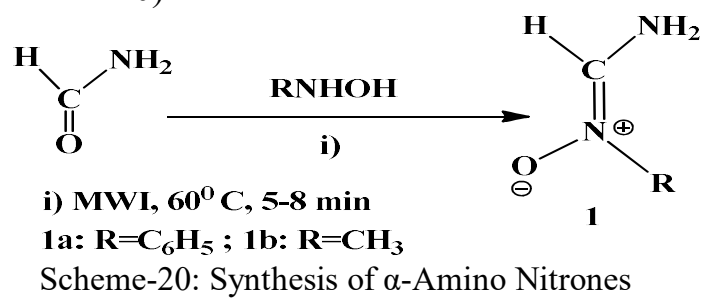

6. Synthesis of bisnitrone from glyoxal in water is also another new concept reported by Chakraborty et. $\mathrm{al}^{25}$ (Scheme-21). This bisnitrone cannot be isolated and cycloaddition reactions are performed insitu.<smiles>O=CC=O</smiles>

Glyoxal

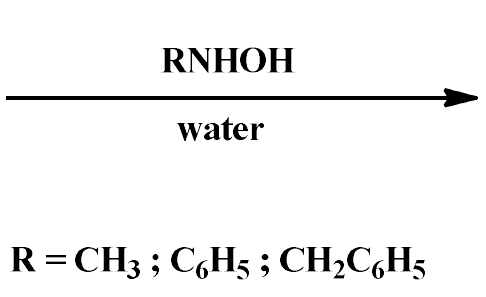

Scheme-21: Synthesis of Bisnitrone in Water<smiles>[R][N+]([O-])=CC([N+]([O-])[O-])=[N+]([R])[O-]</smiles>

1a (Bisnitrone)

\section{Discussion based on Greener Approaches}

\section{Mechanochemical Procedure for the Synthesis and Cycloaddition Reactions of Nitrones}

Among all greener protocols in organic synthesis, a mechanochemical procedure using ball-milling technique has become very popular and attracted the attention of organic chemists in recent years. ${ }^{26}$ This technique is mainly conducted either by hand grinding or by mechanical milling. Hand grinding is usually conducted in a mixer or shaker mill or in a planetary mill at a frequency of 5-60 Hz. The mechanical milling process provides much higher energy and is more sophisticated, reliable than hand grinding procedure.

This is because the latter may lead to varying experimental results which depend on the grinding strength and speed. Sometimes mechanical milling is called grinding in the literature and it may create confusion. So, in order to differentiate between two mechanochemical techniques, it is strongly advised that grinding is defined as the process in a mortar and pestle such as a Retsch RM100 mortar grinder, whereas milling should only refer to that in a mixer or shaker mill or a planetary mill. A mixer or planetary mill can be prepared at home with high speed vibration mill (HSVM) or a commercially available Retsch mixer mill, Spex CertiPrep mixer mill, AGO-2 planetary centrifugal mill etc.

Since mechanical milling in a mixer or planetary mill is always performed with the help of milling balls 
RASĀYAN J. Chem.

Vol. 12 | No. 4 |2183 - 2209| October - December | 2019

therefore, it is often called ball milling process. Among all technical variables like milling frequency, milling time, size and number of milling balls, the material of milling balls, beakers, the first two have been found to be the most important parameters. The efficiency of a chemical reaction is affected when it is conducted in a mixer mill and planetary mill with different working mechanisms. The mechanochemical reactions performed in mixer mill and planetary mill are denoted by MM and PM respectively.

Excellent green chemical aspects like solvent-free reaction, mild conditions, purification of the desired products without chromatography and high yield of products are the important features associated with ball milling mediated reactions. Conducting organic synthesis without disturbing the environment and mankind is always a challenge. So, ball-milling technique may be used as a lucrative methodology in the synthesis of important compounds and this methodology may be used as an alternative to conventional procedures like heating, microwave irradiation and sonication etc. Therefore, synthetic organic chemists should apply mechanochemical procedures like ball-milling as an effective and greener technique for various organic transformations. ${ }^{27}$ Organic synthesis using mechanochemical procedure may involve synthesis of carbon-carbon and carbon-heteroatom covalent bonds, metal-ligand coordination bonds, noncovalent interactions such as hydrogen bonds, halogen bonds or $\pi-\pi$ arene stacking interactions. The mechanochemical procedure and studies on the metal complexes including metal-organic frameworks (MOFs), cocrystallization, inorganic materials, pharmaceutical aspects, supramolecular aspects, and industrial aspects have been already reported in several reviews. ${ }^{26}$ The mechanochemistry of fullerene and its derivatives, a family of unique cage-shaped all-carbon molecules and related materials have been also reported. ${ }^{28}$ It is a serious issue to the organic chemists that the use of toxic chemicals and solvents for chemical transformations is a matter of concern for the environment and mankind. To tackle this growing environmental problem, significant research efforts have been focused on solvent-free reactions. These reactions are often associated with several other advantages such as faster reaction rates, lower energy consumption and easy separation giving rise to products in higher yields and with higher purities. The solvent-free reaction is one of the common techniques employed in ball-milling procedure ${ }^{26}$ which is gradually becoming a powerful tool in the pedagogy of synthetic organic chemistry.

In a typical mechanochemical procedure, reactions are initiated and progressed under frictional force provided either by grinding in a mortar-pestle or by milling in a ball-mill. Recently, mechanochemical synthesis by ball milling has emerged as an effective technique for various organic transformations including "Aldol condensation, ${ }^{29}$ Michael addition, ${ }^{30} \mathrm{Knoevenagel}$ condensation, ${ }^{31}$ Morita-Baylis-Hillman reaction, ${ }^{32}$ Cross-coupling reaction, ${ }^{33} \mathrm{Click}$ reaction, ${ }^{34} \mathrm{Heck}$-type cross-coupling reaction ${ }^{35}$, etc". On the other hand, manual grinding ${ }^{36}$ with a mortar and pestle is generally limited to condensation reactions ${ }^{37}$ including Schiff's base formation, ${ }^{38}$ oxime formation ${ }^{39}$ with occasional exceptions. ${ }^{40}$ However, this a very useful method at the laboratory due to simple and hazardless experimental conditions and is found to be equally effective for the synthesis of heterocyclic compounds of biological interest in last few years. ${ }^{1}$

Amrita Chatterjee et $\mathrm{al}^{41}$ reported an in situ mechanochemical syntheses of nitrones followed by 1,3dipolar cycloaddition reaction leading to chromano isoxazoles (Scheme-22). The reaction was ground for 10-120 minutes followed by heating. An interesting catalyst-free cycloaddition reaction with high yield is the notable feature in this process.

Frederic Lamaty et $\mathrm{al}^{42}$ have reported very interesting results obtained from the solvent-free synthesis of nitrones in a ball-milling procedure. The methodology described in the manuscript is an ideal one for the chemists who want to pursue synthesis on mechanochemistry and studies on reactivity, selectivity as well as use of different solvents for adverse reactivity and yields (Scheme-23).

Yilmaz Yildirir et $\mathrm{al}{ }^{43}$ have developed a fast method for the synthesis of alkyl and aryl- $N$-methylnitrones in a ball-milling technique (Scheme-24 and 25). This simple methodology was widely accepted and has many applications.

\section{1,3-Dipolar Cycloaddition in Ionic Liquids}

With certain excellent properties like good solvating ability, wide liquidious range, tunable polarity, high thermal stability, negligible vapor pressure and ease of recyclability, ionic liquids have emerged as one of the most important green solvents ${ }^{4}$. Hence chemists can perform classical organic reactions in these 
RASĀYAN J. Chem.

Vol. 12 | No. 4 |2183 - 2209| October - December | 2019

media with great advantages in yield and selectivity compared to conventional conditions. Ionic liquids are often referred to as 'designer solvents' for their properties like hydrophilicity, hydrophobicity, lewis acidity, viscosity and density. These properties can be altered by fine-tuning of parameters such as the choice of organic cation, inorganic anion and the length of alkyl chain attached to an organic cation (Scheme-26).

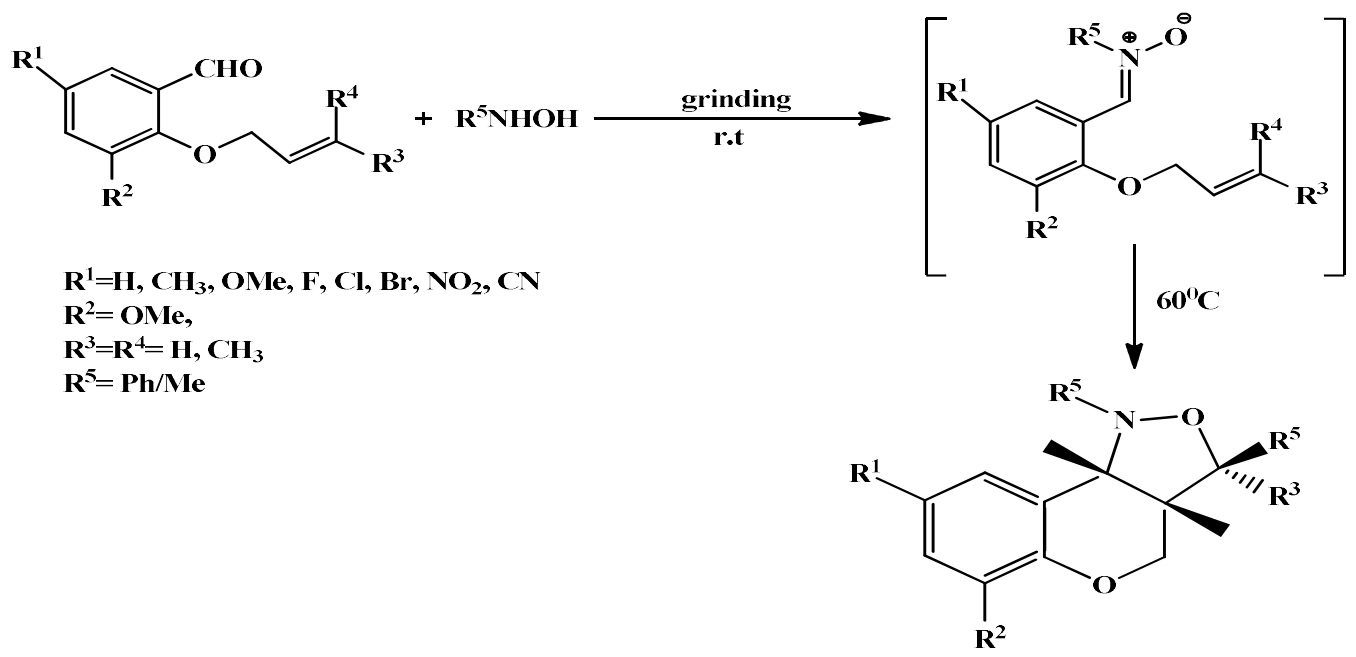

Scheme-22: Mechanochemical Route to cis-Fused Chromano [4,3,-c] Isoxazoles<smiles>C[C+](CCCCC(=O)c1ccccc1)N=Cc1ccccc1</smiles>

Scheme-23: Synthesis of N-Methyl-C-Phenyl Nitrone
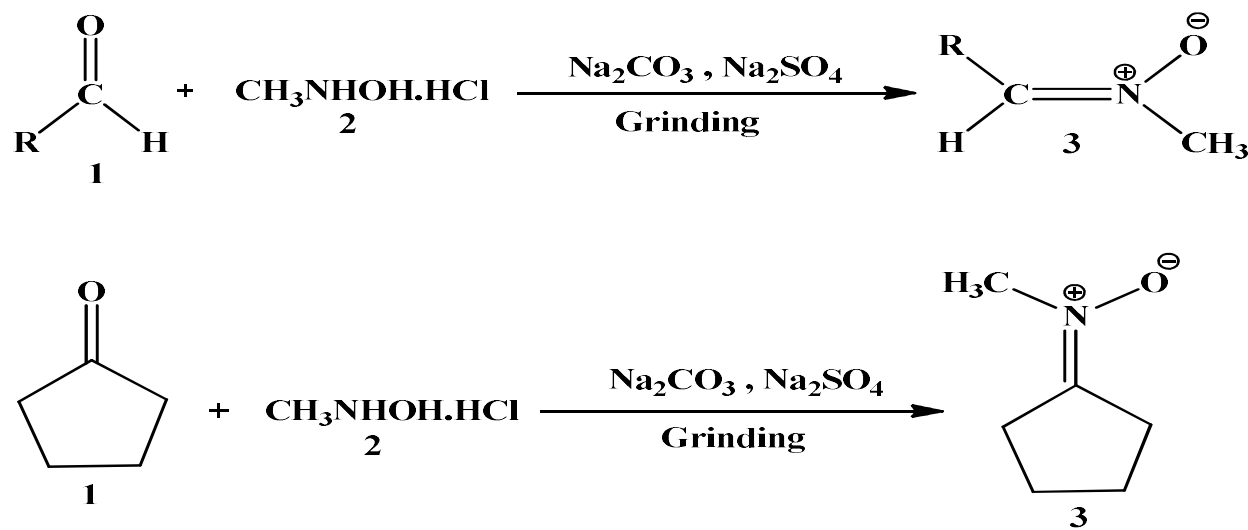

Scheme-24 and 25: Ball-milling Techniques for the Synthesis of Nitrones

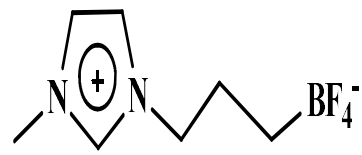

Scheme-26: Chemical Structure of Ionic Liquid 
RASĀYAN J. Chem.

Vol. 12 | No. 4 |2183 - 2209| October - December | 2019

Due to vast structural variations of ionic liquids, working chemists can have plenty of options and flexibility to plan for the methodology of the particular synthesis in this reusable solvent. Due to their composition of non-coordinating ions, ionic liquids can provide an ideal reaction medium for reactions that involve reactive ionic intermediates. Since ionic liquids can have stabilization of charged intermediates, therefore, they can promote unprecedented selectivities and enhanced reaction rates. In addition, ionic liquids are being used as recyclable solvents for the immobilization of transition metal-based catalysts, lewis acids and enzymes ${ }^{45}$. Due to their green credentials and potential to enhance reaction rates and selectivities, ionic liquids have plenty of applications in organic synthesis ${ }^{46}$ with many possibilities for the exploration of newer reactions in ionic liquids. ${ }^{47}$

Kathiravan and Raghunathan ${ }^{48}$ has recently reported an intramolecular 1,3-dipolar cycloaddition reaction in [bmim] $[\mathrm{BF} 4]$ as the recyclable medium and obtained pyrrolo[2,3-a]pyrrolizidino derivatives in good yields (Scheme-27). In order to optimize the method the authors have also carried out the reaction in different organic solvents. The reaction was found to be slower and afforded lower yields of the desired products when performed in conventional organic solvents like methanol, toluene and acetonitrile compared to ionic liquid.

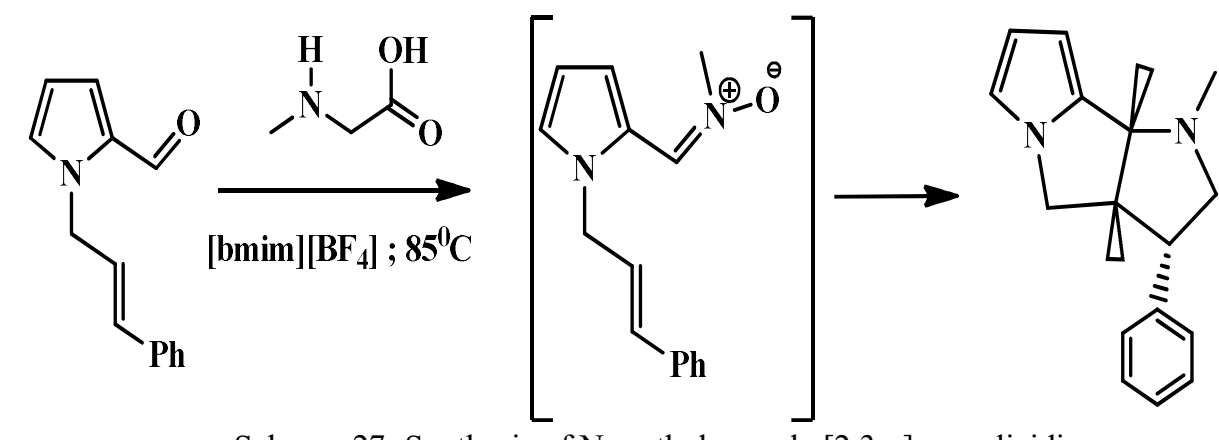

Scheme-27: Synthesis of N-methyl pyrrole [2,3-a] pyrrolizidine

Almansour et $\mathrm{al}^{49}$ have described the regioselective synthesis of dispirooxindolopyrrolidines via 1,3-dipolar cycloaddition reaction in ionic liquid. They have used equimolar ratio of L-phenylalanine and substituted isatins for the synthesis of azomethine ylides. (E)-2-oxoindolino-3-ylidene acetophenone derivative was added in situ to the azomethine ylides in [bmim] $[\mathrm{BF} 4]$ and they have obtained the cycloadducts in $70-77 \%$ yields with high regioselectivity (Scheme-28).

Yadav et $\mathrm{a}^{50}$ have shown that 1-Butyl-3-methylimidazolium based ionic liquids are found to accelerate the reaction rate and yield significantly in the intermolecular 1,3-dipolar cycloaddition reaction of nitrones derived in situ from different aldehydes and phenyl hydroxylamine. They have studied cycloaddition reactions with various electron-deficient alkenes for the synthesis of isoxazolidine derivatives with high regio and diastereoselectivity (Scheme-29). Plenty of applications of this reaction are known.

Bortolini et $\mathrm{al}^{51}$ have shown that 1,3-dipolar cycloaddition reaction of nitrones with alkene ethers affords the corresponding isoxazolidine derivatives in ionic liquid in the presence of $\operatorname{Er}(\mathrm{OTf})_{3}$. The ionic liquid and the catalyst are recycled up to four times without any specific treatment or loss of activity (Scheme-30). This is a very interesting cycloaddition reaction performed in ionic liquid using alkene-ether as active dipolarophile.

Chakraborty et $\mathrm{al}^{52}$ have reported an efficient protocol for the synthesis of a new class of fluoro isoxazolidine and isoxazoline derivatives using $[\mathrm{bmim}] \mathrm{BF}_{4}$ as ionic liquid. The salient feature of this synthesis is excellent diastereoselectivity (Scheme-31). In addition, fluoro nitrone has been successfully used as oxidizing reagent in the synthesis of aldehydes and ketones. This was a new concept of aldehyde and ketone synthesis and the reaction is an atom efficient one.

\section{1,3-Dipolar Cycloaddition in Water}

In the past two decades, organic reactions "in the water" and "on water" have received increased attention because of their environmental acceptability, abundance and low cost. ${ }^{53,54,55}$ In addition, water has also some unique reactivity and selectivity which are not observed or can be obtained in conventional organic 
RASĀYAN J. Chem.

Vol. 12 | No. 4 |2183 - 2209| October - December | 2019

solvents. ${ }^{56,57,58}$ Therefore, development of efficient chemical transformations and newer synthesis in water without any catalyst is highly accepted and appreciated. It has been observed that aqueous phase cycloaddition reaction produces a number of new heterocycles with regio and stereoselectivity peculiarity. Isoxazolidines, isoxazoles and pyrrolidines are examples of this class of heterocycles. There are many factors on which the rate of acceleration of organic reactions in aqueous media depends. ${ }^{53,54}$ These include high cohesive energy, density of water, the high internal pressure within the medium, the hydrogen-bonding ability, the hydrophobic packing of diene and dienophile in cycloaddition reactions, the hydrophobic vs. antihydrophobic effects, the micellar catalysis, the solvophobicity, and the solvent polarity. The concept of "on-water" reactions ${ }^{59,60}$ was first introduced by Sharpless in 2005. In "on water" method the reactants with water are kept stirring to generate an aqueous suspension and it has been observed that both kinetics and yields are extremely increased in most cases compared to those in organic solvents.<smiles>[R4]c1ccc2c(c1)C(=O)C(=O)N2</smiles>

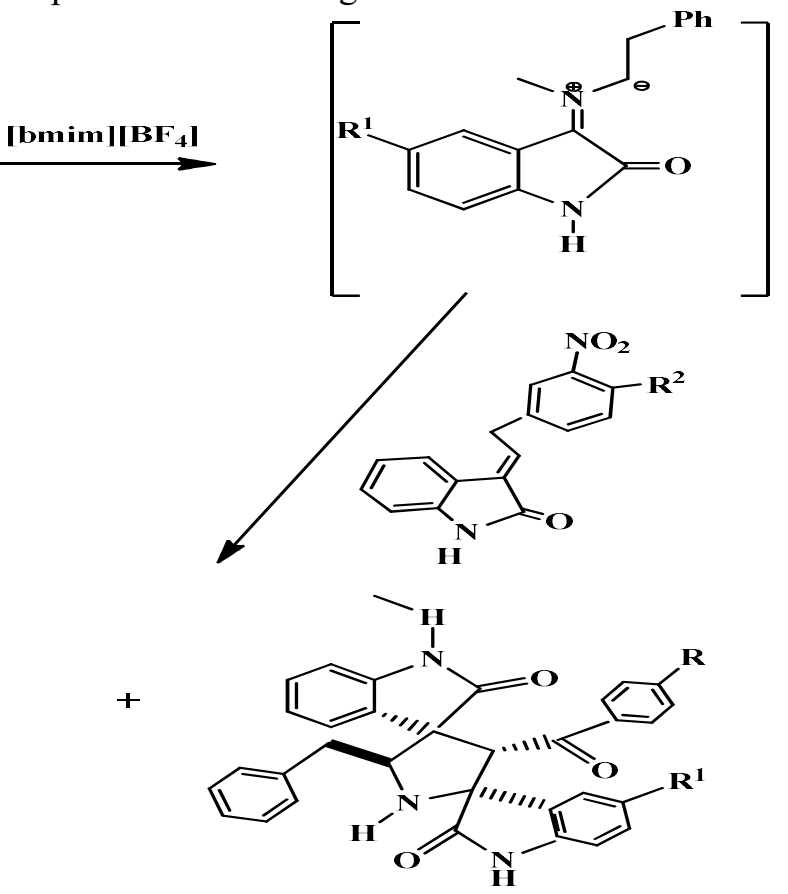

B $7 \%$

Scheme-28: Synthesis of di spirooxindolo pyrrolidines

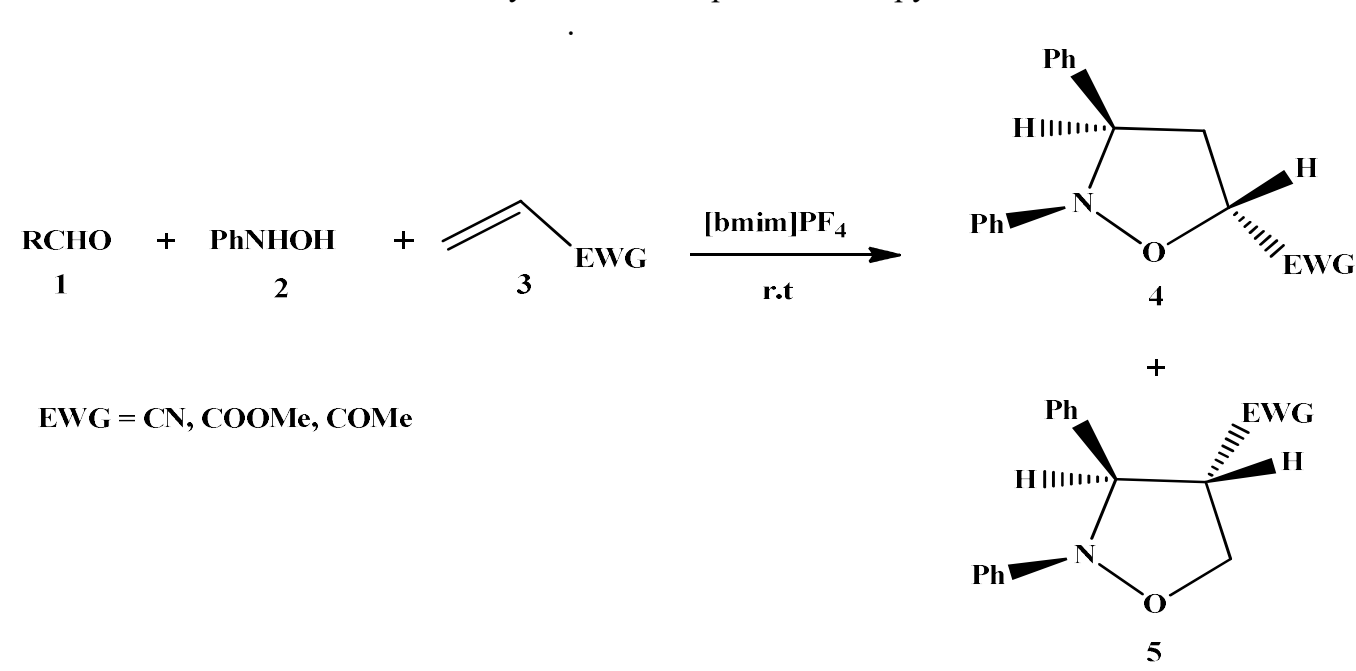

Scheme-29: Ionic Liquid mediated Regioselective Synthesis of Isoxazolidines 
RASĀYAN J. Chem.

Vol. 12 | No. 4 |2183 - 2209| October - December | 2019

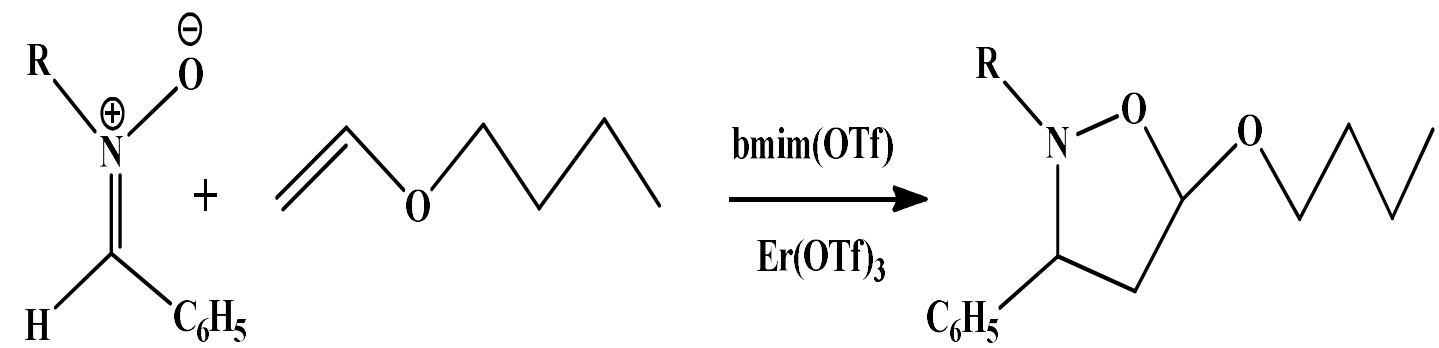

$\mathrm{R}=\mathrm{CH}_{2} \mathrm{C}_{6} \mathrm{H}_{5} ; \mathrm{CH}_{3}$

Scheme-30: Ionic Liquid and Catalyst mediated Synthesis of Isoxazolidines

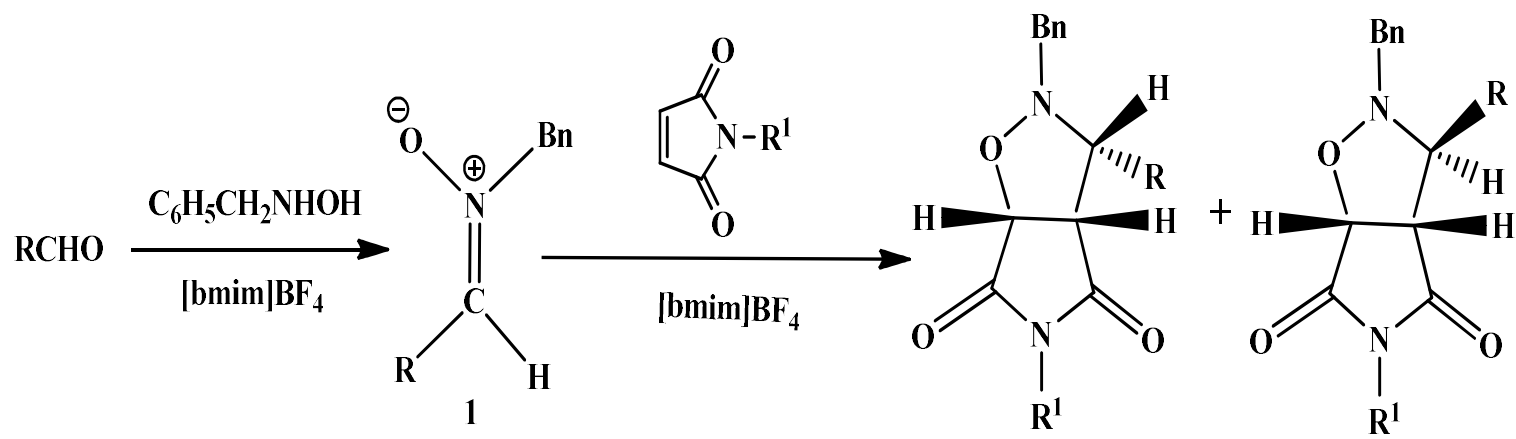

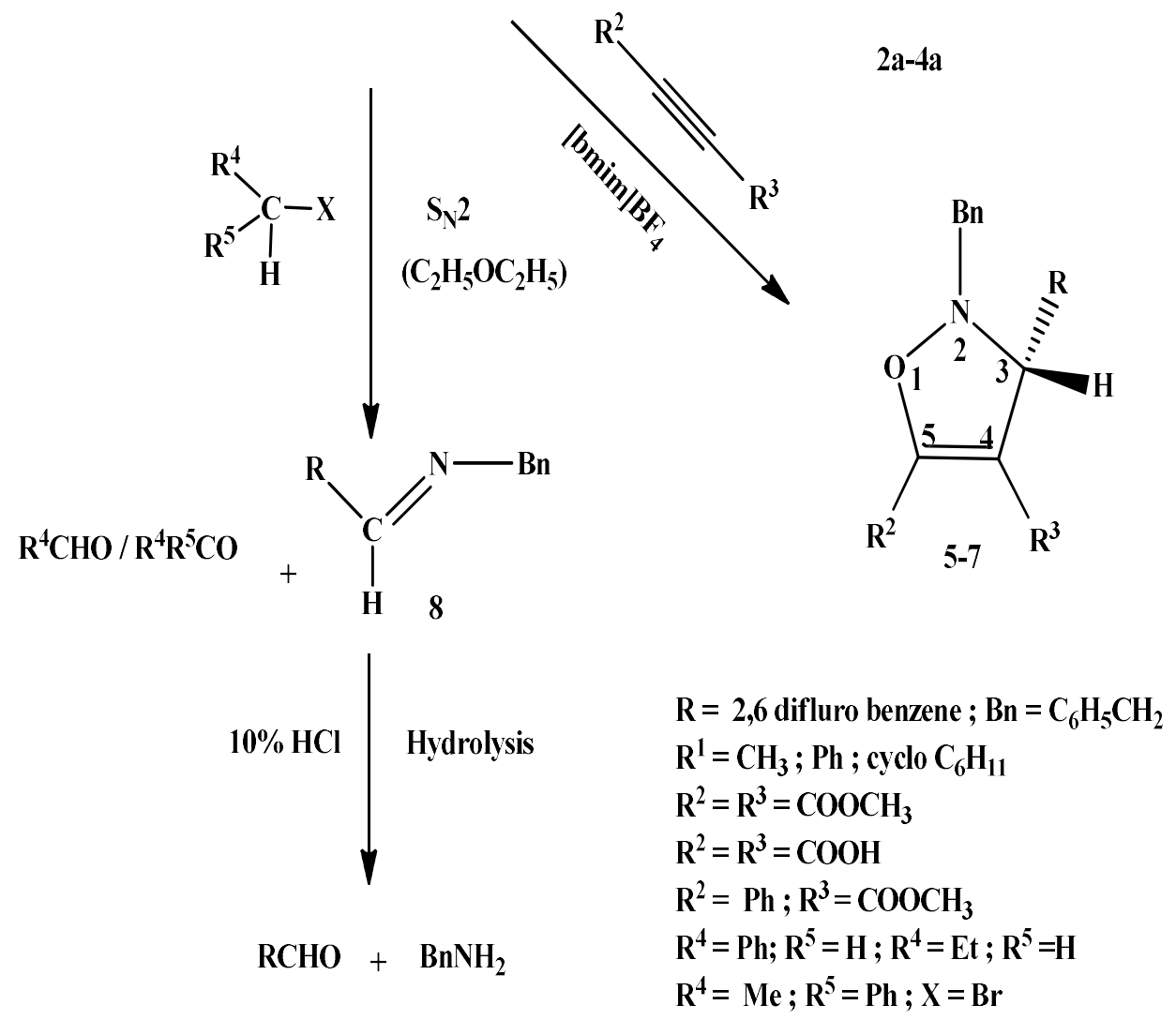

Scheme-31: Synthesis of Fluoro Isoxazoline and Isoxazolidine Derivatives using Fluoro Nitrone and Application of the Nitrone in Atom efficient Aldehyde and Ketone Synthesis 
RASĀYAN J. Chem.

Vol. 12 | No. 4 |2183 - 2209| October - December | 2019

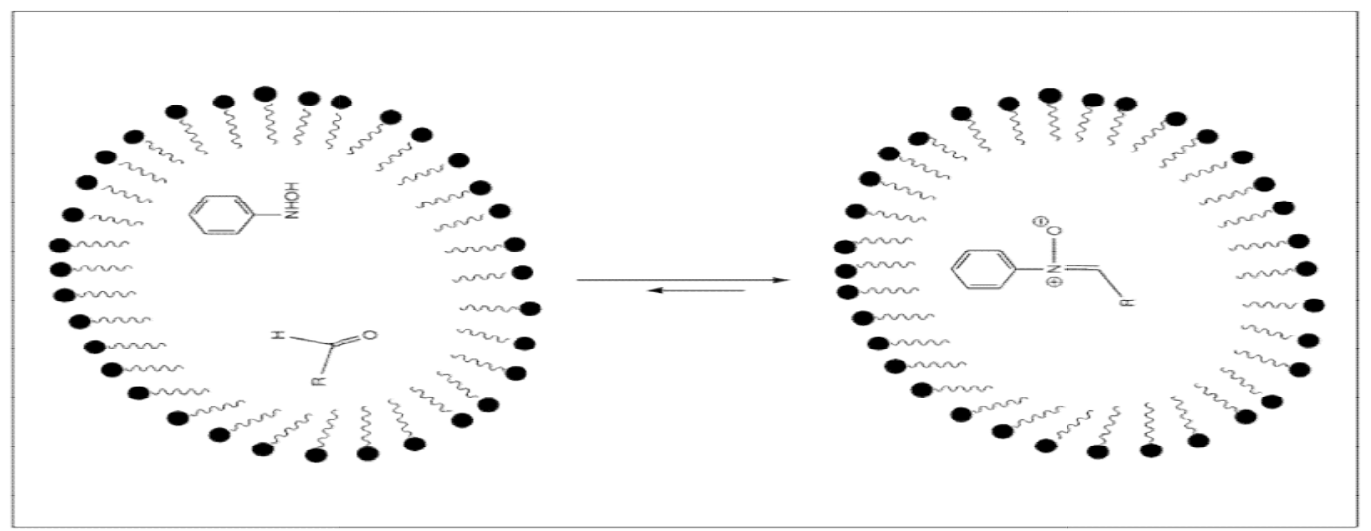

Scheme-32: Synthesis of Nitrone on Water

Plenty of interesting reports are found in the literature based on aqueous phase cycloaddition reactions. A few interesting works are included in this review work. Butler et $\mathrm{al}^{61}$ have reported an interesting study on the influence of water on the rates of 1,3-dipolar cycloaddition reactions. The work is very much helpful to the scientists who intend to work in aqueous phase synthesis of nitrone cycloaddition reactions and also the effects of water in these reactions (Scheme-33).

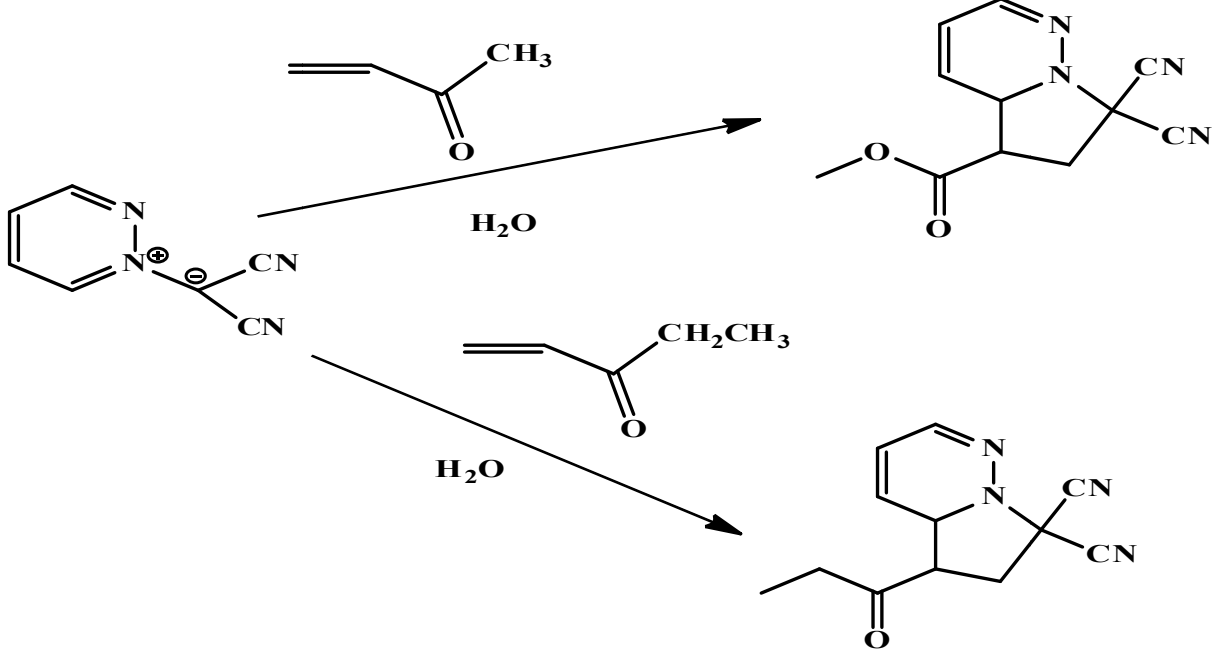

Scheme-33: Influence of Water on the Rates of Cycloaddition Reaction

An interesting example of the formation of nitrone in water exclusion reaction in water using surfactant and subsequent cycloaddition reactions in the same pot have been reported by P. K. Bhattacharya et al. ${ }^{62}$ This is a new concept in aqueous phase synthesis and has many applications (Scheme-34).

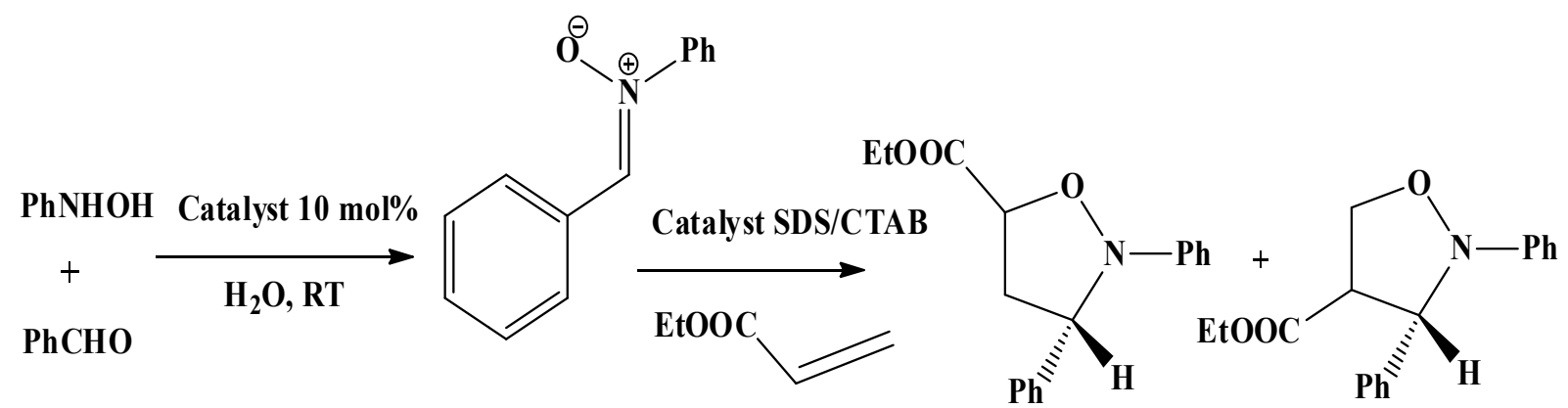

Scheme-34: Formation of Nitrone in Water Exclusion Reaction in Aqueous Media Using Surfactant 
RASĀYAN J. Chem.

Vol. 12 | No. 4 |2183 - 2209| October - December | 2019

Pedro de Armas et $\mathrm{al}^{63}$ have reported the first example of a regioselective organocatalyzed 1,3-dipolar cycloaddition reaction between conjugated alkynoates and nitrones "on water" (Scheme-35). This work may be very much helpful to the organic chemists who intend to perform "on water" synthesis.

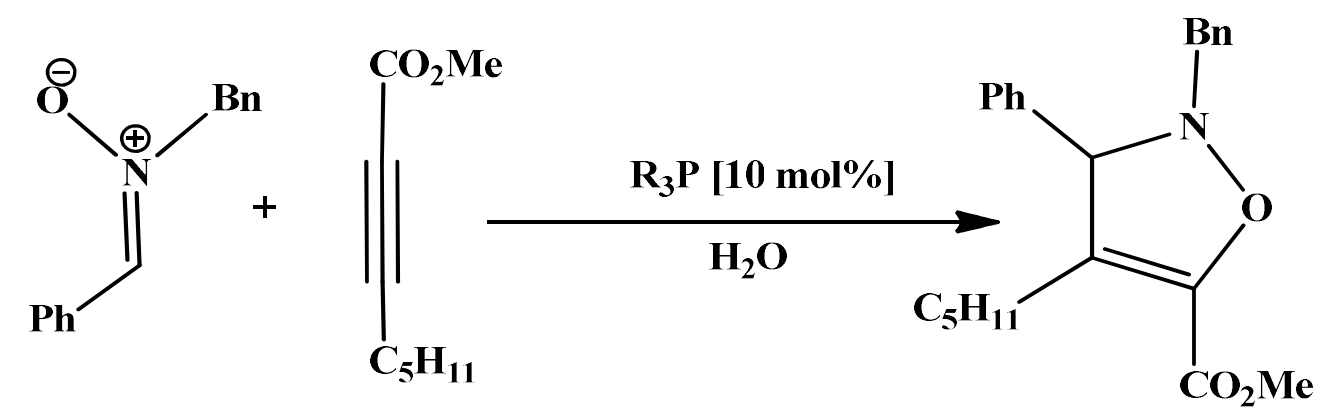

Scheme-35: Regioselective 1,3-Dipolar Cycloaddition Reaction Between Conjugated Alkynoates and Nitrones on Water

Synthesis of $\beta$-lactum using Kinugasa reaction is one of the pioneering discoveries in nitrone cycloaddition reactions by Basak et al. Recently, Kinugasa reaction has been explored by a number of research groups. ${ }^{64,65}$ "On water" approach to this reaction has been successfully investigated. Usually, Kinugasa reactions are performed in organic solvents like $\mathrm{DMF}, \mathrm{MeCN}$ and sometimes in neat pyridine. Basak et al have reported successful Kinugasa reaction with copper catalyst in aqueous conditions like DMF/water, t$\mathrm{BuOH} /$ water and $\mathrm{MeCN} /$ water respectively. ${ }^{66}$ The authors have also performed the reaction in pure water. However, the efficiency and yield were decreased. Recently, the first asymmetric Kinugasa reaction has been performed "on water". ${ }^{67}$ Introduction to chirality was achieved in the reaction using a C2-symmetric secondary diamine ligand to coordinate the copper species. The optimized reaction conditions include $\mathrm{nBu}_{2} \mathrm{NH}$ as the base, copper (II) triflate as the copper source and water as the solvent at a reaction temperature of $20^{\circ} \mathrm{C}$ (Scheme-36).

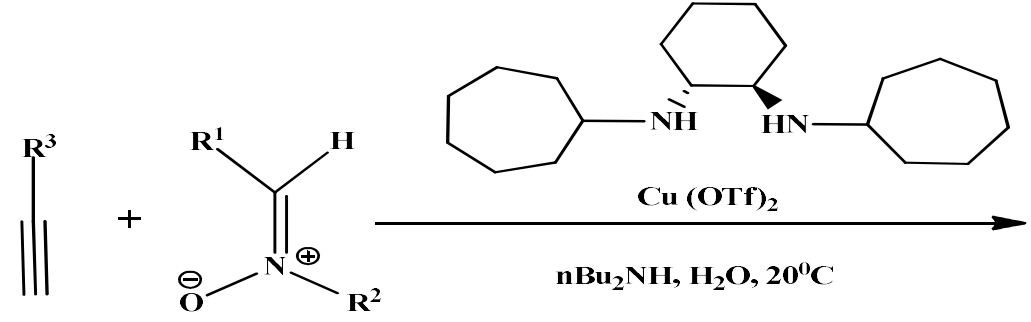

Reagents $\&$ conditions:

Cu $(\text { OTf })_{2}(10 \mathrm{~mol} \%)$, Ligand (20 mol\%), alkyne (0.2 mmol),

nitrone (0.21 mmol), nBu $2 \mathrm{NH}(0.2 \mathrm{mmol})$, water $(0.4 \mathrm{~mL}), 20^{0} \mathrm{C}$

Scheme-36: Enantioselective beta-lactum Synthesis Under Aqueous Conditions
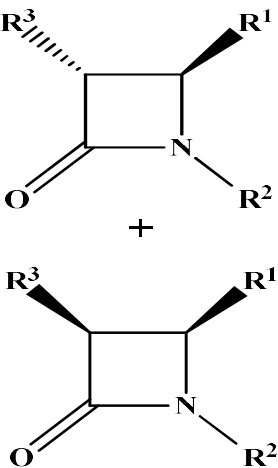

Chakraborty et $\mathrm{al}^{68}$ have also reported some interesting results in aqueous phase synthesis using $\alpha$-chloro nitrone. The nitrone was synthesized for the first time in the aqueous phase and the cycloaddition reactions showed excellent diastereoselectivity (Scheme-37).

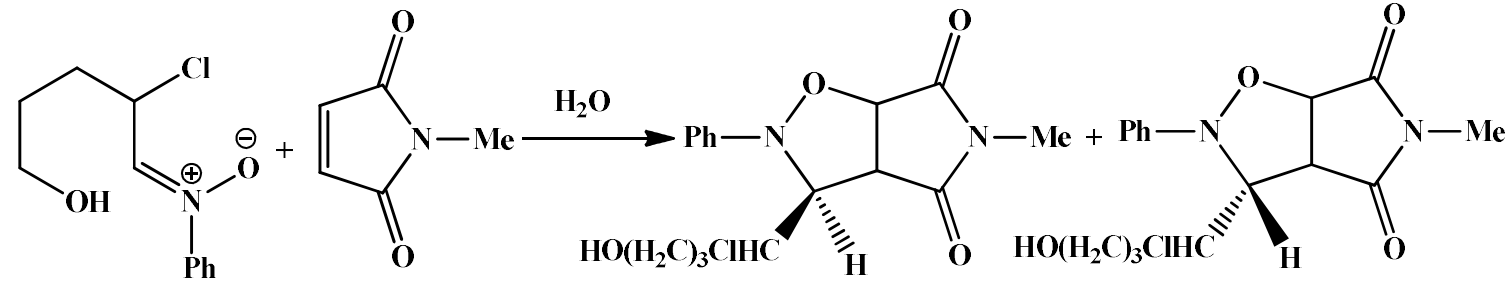

Scheme-37: Diastereoselective Synthesis of Isoxazolidines Using Alfa Chloro Nitrone in Aqueous Phase 
RASĀYAN J. Chem.

Vol. 12 | No. 4 |2183 - 2209| October - December | 2019

Chakraborty et $\mathrm{al}^{69}$ have also reported the synthesis of new bisisoxazolidine derivatives using glyoxal derived bisnitrone with various activated dipolarophiles in water (Scheme-38). This is a new concept of synthesis of bisnitrones from glyoxal in water.<smiles>[R][N+]([O-])=CC(C)=[N+]([R])[O-]</smiles>

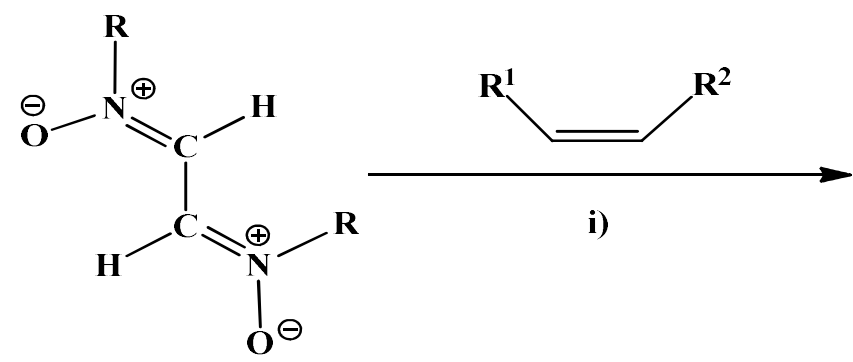

1 (Bisnitrone)

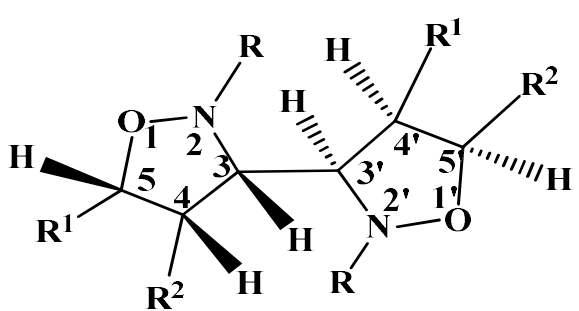

2 - 6 (Bisisoxazolidine derivatives)

i) water, $\mathrm{RT}, 3-5 \mathrm{hr}, \mathrm{N}_{2}$ atmosphere

$\mathrm{R}=\mathrm{CH}_{3} ; \mathrm{C}_{6} \mathrm{H}_{5} ; \mathrm{CH}_{2} \mathrm{C}_{6} \mathrm{H}_{5}$

$2: R^{1}, R^{2}=$-CONMeCO-

$3: \mathbf{R}^{1}, \mathbf{R}^{2}=-\mathrm{CONPhCO}-$

$4: R^{1}, R^{2}=-C O N C y C O-$

$5: \mathbf{R}^{1}=\mathrm{H} ; \mathbf{R}^{2}=-\mathrm{CO}_{2} \mathrm{CH}_{3}$

$6: \mathrm{R}^{1}=\mathrm{H} ; \mathrm{R}^{2}=-\mathrm{C}_{6} \mathrm{H}_{5}$

Scheme-38: Synthesis of Bisnitrones and Bisisoxazolidine Derivatives From Glyoxal in Water

\section{1,3-Dipolar Cycloaddition Under Microwave Irradiation}

The use of microwaves in organic synthesis has become an indispensable tool ${ }^{70}$ in the last two decades. In recent years, microwave-induced reactions are found to be one of the most favorable choices in organic synthesis. This is because by employing this technology it is generally possible to prepare organic compounds very quickly with high purity and good yields compared to other more conventional methods. ${ }^{71}$ Moreover, microwave technology is also considered as an important approach towards 'Green Chemistry' because of its eco-friendly nature. Therefore, using microwave irradiation a variety of isoxazolidine and isoxazoline derivatives can be synthesized in a shortened reaction time with good to excellent yields. ${ }^{53}$

Pineiro Melo et $\mathrm{al}^{70}$ in their exclusive review on "Microwave-Assisted 1,3-Dipolar Cycloaddition: an EcoFriendly Approach to Five-Membered Heterocycles" stated that "this non-conventional energy source is not only reduced chemical reaction times, but increase yields and in some cases can lead to different outcomes from those obtained with conventional heating".

Loupy et $\mathrm{a}^{72}$ have first reported microwave-induced nitrone cycloaddition reaction, where they have shown the utility of focused microwaves as energy source in the 1,3-dipolar cycloaddition reactions of $N$-methyl$\alpha$-phenyl nitrones with fluorinated dipolarophiles under solvent-free condition leading to isoxazolidine derivatives having biological activities. Under classical heating, they performed the reactions with refluxing in toluene $\left(110^{\circ} \mathrm{C}\right)$ with limited yields after long times. Both the yields and experimental conditions were improved in solvent-free conditions and even more under microwave irradiation (Scheme-39). This is also interesting to mention that regio and stereoselectivities remain unchanged irrespective of the experimental 
RASĀYAN J. Chem.

Vol. 12 | No. 4 |2183 - 2209| October - December | 2019

condition. $^{72}$ Loupy et al have also reported an interesting change in yield for the synthesis of fluoro cycloadducts (Scheme-40).<smiles>C[N+]([O-])=Cc1ccccc1</smiles>

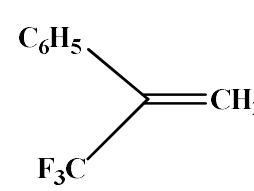

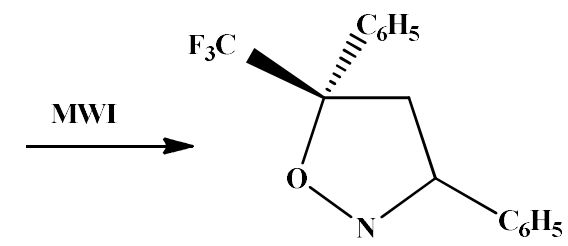

$\begin{array}{lllll}\text { MWI } & \text { no Solvent } & 3 \mathrm{~min} & 170^{\circ} \mathrm{C} & 98 \% \\ \triangle & \text { no Solvent } & 3 \mathrm{~min} & 170^{\circ} \mathrm{C} & 55 \% \\ \triangle & \text { no Solvent } & 48 \mathrm{hrs} & 110^{\circ} \mathrm{C} & \mathbf{6 5 \%}\end{array}$

Scheme-39: Microwave induced 1,3-Dipolar Cycloaddition of N-Methyl-alfa-phenyl nitrone with Fluorinated Dipolarophile under Solvent-Free Condition

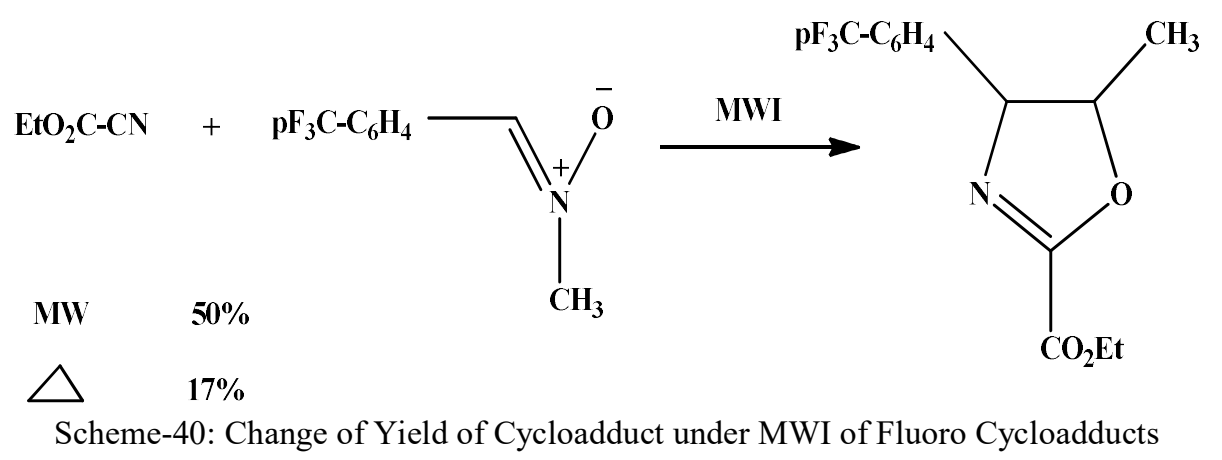

Antonio de la Hoz et $\mathrm{al}^{73}$ have also reported many aspects of microwave-induced reactions in their review "Cycloadditions under Microwave Irradiation Conditions: Methods and Applications". From the review it has been noted that microwave-induced cycloaddition reactions are capable of exploring new dimensions not only in the changes in chemo, regio and diastereoselectivity but also in the transition state of the reactions as well. The review also reports synthesis of new heterocyclic compounds by cycloaddition reactions with good to excellent yields and high selectivity. The review also reported about those cycloaddition reactions which are very reluctant to participate in conventional procedures, such as synthesis of pyrazoles. The reaction can be performed under microwave irradiation conditions. In most cases, wonderful accelerations and great improvements in yields and reaction conditions have been observed. Microwave induced reactions thus open up new possibilities for modifying the result of competitive reactions by considering the relative hardness of the transition states.

Dujardin et $\mathrm{al}^{74}$ have reported a highly efficient solvent-free 1,3-dipolar cycloaddition reaction of $\mathrm{N}$ substituted dipolarophiles and nitrones. New isoxazolidine derivatives were synthesized in good to excellent yields by 1,3-dipolar cycloaddition reactions of $N$-vinyl amide dipolarophiles and nitrones. Strikingly solvent-free conditions gave high conversion and yields in shortened reaction time and minimized degradation products (Scheme-41).

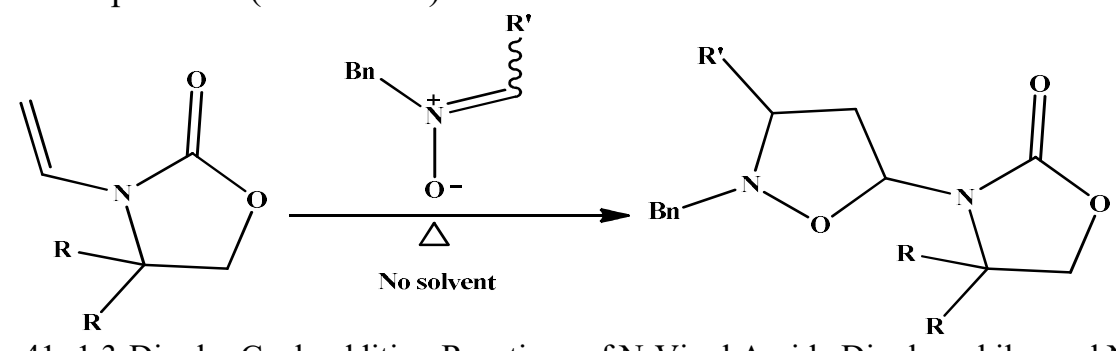

Scheme-41: 1,3-Dipolar Cycloaddition Reactions of N-Vinyl Amide Dipolarophiles and Nitrones with Excellent Yield 
RASĀYAN J. Chem.

Vol. 12 | No. 4 |2183 - 2209| October - December | 2019

Chakraborty et $\mathrm{al}^{75}$ have reported some interesting observations in nitrone cycloaddition reactions under microwave-induced reactions. Cycloaddition reactions of bisnitrone with electron-deficient alkynes developed bis-isoxazoline derivatives. Further, they were converted into synthetically more important aziridine derivatives successfully (Scheme-42). This reaction has many applications.

Chakraborty et $\mathrm{al}^{76}$ have also successfully reported the synthesis of spiro isoxazolidine derivatives under microwave-induced 1,3-dipolar cycloaddition reactions of $\alpha$-chloro nitrone in atom efficient reactions with excellent yields (Scheme-43).

\section{Light-induced Cycloaddition Reactions}

Aza-heterocycles are one of the important classes of compounds due to their potential biological activities. They are usually obtained in natural products and also used as drugs. There are many methods developed for the synthesis of 1,2,4-oxadiazolines and these procedures are carried out using [4+2] cycloaddition reaction of an imine with a nitrile oxide. The nitrile oxides are usually generated in situ from hydroxamoyl chloride or nitroalkane. Since the existing general procedures for the synthesis of 1,2,4-oxadiazolines needs drastic reaction conditions, therefore, greener methods for their synthesis are highly desirable. Soni et $\mathrm{al}^{77}$ have very recently presented a greener method for the synthesis of 1,2,4-oxadiazolines via an intramolecular oxidative cyclisations of amidoximes in the presence of an organocatalyst and molecular oxygen. The authors optimized the reaction conditions to give 3-phenyl-5,6,7,7a-tetrahydropyrrolo[1,2d] $[1,2,4]$ oxadiazole from phenyl(pyrrolidin-1-yl)methanone oxime. They optimized the reaction conditions using $2 \mathrm{~mol} \%$ of an organophotocatalyst, 2,4,6- tris(4-fluorophenyl)pyrylium tetrafluoroborate [T(pF)PPT] at a $0.2 \mathrm{M}$ concentration in DMF under an atmosphere of molecular oxygen. Visible-light irradiation was provided by a compact fluorescent lamp (CFL, $23 \mathrm{~W})$. Organophotocatalyst can reduce the drawbacks of transition metals related to toxicity (Scheme-44).<smiles>O=Cc1ccc(C=O)cc1</smiles>

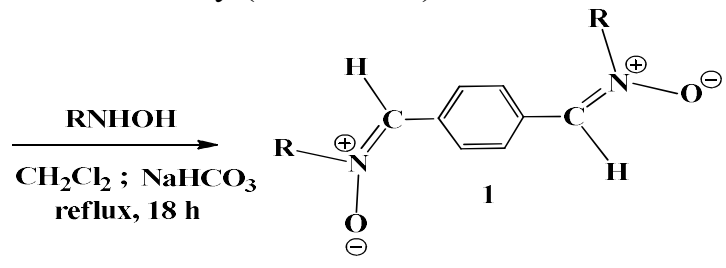

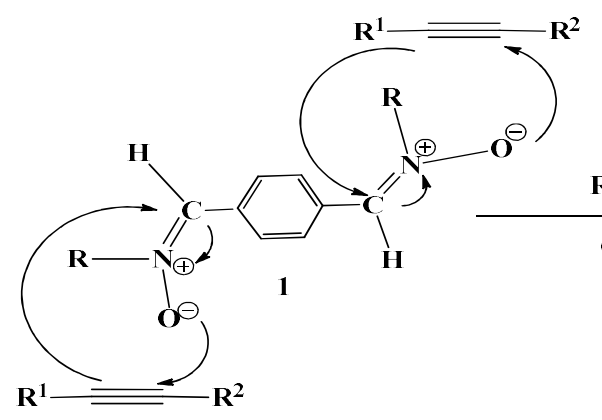

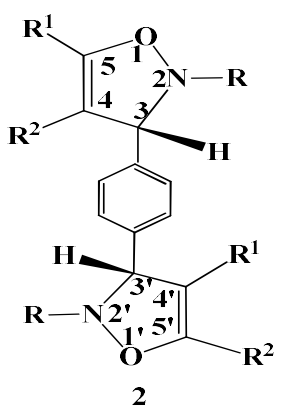

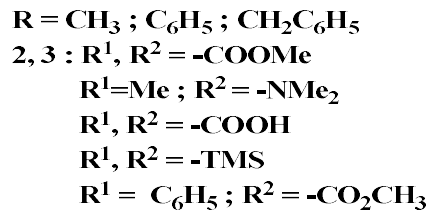<smiles></smiles>

Scheme-42: Synthesis of Bisisoxazoline and Bisaziridine Derivatives 
RASĀYAN J. Chem.

Vol. 12 | No. 4 |2183 - 2209| October - December | 2019<smiles>[R]NC(C)=C1CCCO1</smiles>

$\mathrm{R}=\mathrm{CH}_{3} ; \mathrm{C}_{6} \mathrm{H}_{5} ; \mathrm{C}_{6} \mathrm{H}_{5} \mathrm{CH}_{2} ; \mathrm{C}_{6} \mathrm{H}_{11}$

Scheme-43: Microwave-Assisted Synthesis of Novel spiro isoxazolidines

Reaction Condition: (i) MWI, DMF (2 mL), 5-10 min, 34-40 $\mathrm{C}$<smiles>O/N=C(/c1ccccc1)N1CCCC1</smiles>

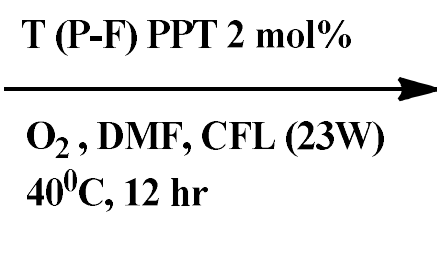<smiles>c1ccc(C2=NOC3CCCN23)cc1</smiles>

Scheme-44: Synthesis of Bicyclic 1,2,4-Oxadiazolines in Light-induced Reactions

Mekheimer et $\mathrm{a}^{78}$ have reported a new technique of cycloaddition reaction involving the reaction between nitrones and acetonitrile derivatives under solar heating efficiently leading to isoxazolidines which further underwent rearrangement to unexpected 3-aryl-2-cyanothioacrylamide and 3-aryl-2-(hetaryl)acrylonitrile derivatives. The procedure has significant advantages over conventional methodologies viz, greener approach, high yielding and simple work-up procedure (Scheme-45).

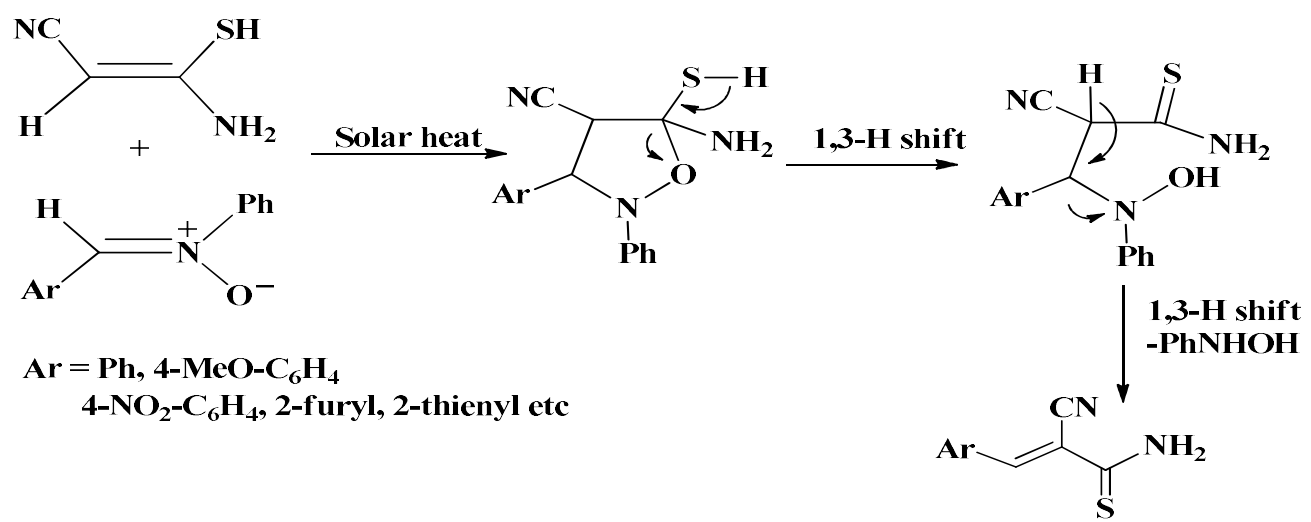

Scheme-45: Reaction between Nitrones and Acetonitrile Derivatives under Solar Heating for the Synthesis of Isoxazolidines

\section{CONCLUSION}

We have tried to represent the latest developments in 1,3-dipolar cycloaddition reactions with special emphasis on greener approaches. We have incorporated the various synthetic procedures of nitrone synthesis and also 1,3-dipolar cycloaddition reactions based on green chemistry methodologies. It has been observed that many reviews are available on nitrone cycloaddition reactions in literature but reviews with special emphasis on the application of greener methodologies are rare. Therefore, we believe this account on nitrone cycloaddition reactions will help the researchers to a great extent especially who intends to work on green synthesis of nitrone and cycloaddition reactions. 
RASĀYAN J. Chem.

Vol. 12 | No. 4 |2183 - 2209| October - December | 2019

\section{ACKNOWLEDGMENT}

The author is grateful to Department of Biotechnology, Ministry of Science \& Technology, Government of India, New Delhi for providing Overseas Associateship-Fellowship (Grant no: BT/20/NE/2011) and also administrative support and help for conducting the research work at the School of Chemistry, Cardiff University, Park Place, Wales, UK.

\section{REFERENCES}

1. (a) J. J. Tufariello,1,3-Dipolar Cycloaddition Chemistry, Edited by A. Padwa (Wiley: New York) 1984, DOI: 10.1002/jhet.5570230658; (b) K.B.G. Torssell, Nitrile Oxides, Nitrones and Nitronates in Organic Synthesis, (VCH: New York) 1988, DOI: 10.1002/ange.19891010245; A. Padwa and W.H. Pearson, Synthetic Application of 1,3-Dipolar Cycloaddition Chemistry toward Heterocycles and Natural Products, (Wiley-New Jersey) 2003, DOI: 10.1002/0471221902

2. W. Patterson, P.S. Cheung and M. J. Ernest, J. Med. Chem., 35, 507(1992), DOI: 10.1021/jm00081a011

3. E. Wagner, L. Becan and E. Nowakowska, Bio. Org. Med. Chem., 12, 265(2004), DOI: 10.1016/j.bmc.2003.10.004

4. K.N. Houk, J. Sims and C. R. Lukas, J. Am. Chem. Soc.,95, 7301(1973), DOI: 10.1021/ja00803a018

5. R. Sustman,Pure. Appl. Chem., 40, 569 (1974), DOI: 10.1351/pac197440040569

6. R. Huisgen,J. Org. Chem., 41, 403 (1976), DOI: 10.1021/jo00865a001

7. S. Kanemasa and O. Tsuge, Heterocycles, 30, 719 (1990).

8. R.B. Woodward and R. Hoffmann, The Conservation of Orbital Symmetry (Verlag Chemie, Weinheim), 1970, DOI: 10.1002/anie.196907811

9. K.V. Gothelf, R.G. Hazell and K.A. Jorgensen, J. Org. Chem., 61, 346(1996), DOI: $10.1021 /$ jo951204e

10. I.A. Grigor'ev and H. Feuer, Ed. Nitrile Oxides, Nitrones, and Nitronates in Organic Synthesis. Nitrones: Novel Strategies in Synthesis (Wiley-Interscience, Hoboken, NJ), 2, 129-434 (2008), DOI: 10.1002/9780470191552.ch2

11. K.N. Houk and K. Yamaguchi, 1,3-Dipolar Cycloaddition Chemistry(Wiley, New York),2, 407 (1984), DOI: 10.1002/jhet.5570230658

12. K.N. Houk, Top. Curr. Chem,79, 1(1979)

13. (a) J. Hamer and A. Macaluso, Chem. Rev.,64, 473(1964), DOI: 10.1021/cr60230a006; (b) I. L. Smith, Chem. Rev, 23, 193(1938), DOI: 10.1021/cr60075a001

14. (a) P. Merino, T. Tejero, I. Delso and R. Matute, Org. Biomol. Chem, 15, 3364(2017), DOI: 10.1039/C7OB00429J; (b) R. Matute, S. Garcia-Viñuales, H. Hayes, M. Ghirardello, A. Daru, T. Tejero, I. Delso and P. Merino, Curr. Org. Synth., 13, 669 (2016); (c) M. Lombardo and C. Trombini, Synthesis, 759 (2000).

15. (a)S.I. Murahashi and Y. Imada, Chem. Rev., 119, 4684(2019), DOI: 10.1021/acs.chemrev.8b00476; (b)F.Cardona and A. Goti A, Angew. Chem. Int. Ed., 44, 832(2005), DOI: 10.1002/anie.200502640

16. G. Masson, S. Py and Y. Vallee, Angew. Chem. Int. Ed, 41, 1772(2002), DOI: 10.1002/15213773(20020517)41:10\%3C1772::AID-ANIE1772\%3E3.0.CO;2-Q

17. (a) K. Folting, W.N. Lipscomb and B. Jerslev, Acta. Chemica. Scandinavica, 17, 2138 (1963), DOI: 10.3891/acta.chem.scand.17-2138; (b) K. Folting, W.M. Lipscomb and B. Jerslev, Acta. Cryst, 17, 1263 (1964), DOI: 10.1107/S0365110X6400319X

18. J N. Brown and L.M. Trefonas, Acta. Cryst, B29, 237(1973), DOI: 10.1107/S0567740873002268

19. P. T. Anastas and J. C. Warner, Green Chemistry: Theory and Practice (Oxford University Press, UK), 2000

20. J. Moran, Y. Pfeiffer, S.I. Gorelsky and A.M. Beauchemin, Org. Lett., 11,1895(2009), DOI: $10.1021 / 01900292 \mathrm{r}$

21. Q. Zeng, L. Zhang, J. Yang, B. Xu, Y. Xiao and J. Zhang, Chem. Commun., 50, 4203(2014), DOI: $10.1039 / \mathrm{C} 4 \mathrm{CC} 00493 \mathrm{~K}$

22. I. Nakamura, T. Onuma, R. Kanazawa, Y. Nishigai and M. Terada, Org. Lett., 16, 4198 (2014), DOI: $10.1021 / \mathrm{ol} 501889 \mathrm{~g}$

23. P. De March, M. Figueredo, J. Font, S. Milán, A. Alvarez-Larena, J.F. Piniella and E. Molins, 
RASĀYAN J. Chem.

Vol. 12 | No. 4 |2183 - 2209| October - December | 2019

53,2979(1997), DOI: 10.1016/S0040-4020(96)01177-5

24. B. Chakraborty and N.Rai, J. Indian. Chem. Soc., 89 (5), 671(2012).

25. B. Chakraborty and G.P. Luitel, J. Heterocyclic. Chem., 52, 726(2015), DOI: 10.1002/jhet.2181

26. (a) B.C. Ranu and A. Stolle, Ball Milling Towards Green Synthesis: Applications, Projects and Challenges (Royal Society of Chemistry, UK), 2015; (b) I. Huskic, I. Halasz, T. Friscic and H. Vancik, Green Chem, 14, 1597(2012), DOI: 10.1039/C2GC35410A

27. (a) J.G. Hernandez and E. Juaristi, J. Org. Chem.,76, 1464(2011), DOI: 10.1021/jo1022469; (b) J.G. Hernandez , V. Garcia-Lopez and E. Juaristi, Tetrahedron, 68, 92(2012), DOI: 10.1016/j.tet.2011.10.093; (c) Y.F. Wang, R.X. Chen, K. Wang, B.B. Zhang, B.Z. Lib and D.Q. Xu, Green. Chem., 14, 893 (2012); (d) M. Jorres, S. Mersmann, G. Raabe and C. Bolm, Green. Chem., 15, 612 (2013); (e) R. Trotzki, O. Hoffmann and B. Ondruschka, Green. Chem., 10, 873 (2008); (f) J. Mack and M. Shumba, Green. Chem., 9, 328 (2007); (g) D.C. Waddell and J. Mack, Green. Chem., 11, 79 (2009); (h) F. Schneider, T. Szuppa, A. Stolle, B. Ondruschka B and H. Hopf, Green. Chem., 11, 1894 (2009); (i) G. Cravotto, D. Garella, S. Tagliapietra, A. Stolle, S. Schusler, S.E.S. Leonhardt and B. Ondruschka, New. J. Chem., 36, 1304 (2012); (j) D.A. Fulmer, W.C. Shearouse, S.T. Medonza and J. Mack, Green. Chem., 11, 1821 (2009); (k) R. Thorwirth, A. Stolle and B. Ondruschka, Green. Chem., 12, 985 (2010); (1) R. Thorwirth, A. Stolle, B. Ondruschka, A. Wild and U.S. Schubert, Chem. Commun., 47, 4370 (2011); (m) T.L. Cook, J.A. Walker and J. Mack, Green. Chem., 15, 617 (2013); (n) V. Estevez, M. Villacampa and C.J. Menendez, Chem. Commun., 49, 591 (2013); (o) W. Su, J. Yu, Z. Li and Z. Jiang, J. Org. Chem., 76, 9144 (2011); (p) V. Strukil, B. Bartolec, T. Portada, I. Đilovic, I. Halasz and D. Margetic, Chem. Commun., 48, 12100 (2012); (q) G.J. Hernandez and E. Juaristi, J. Org. Chem.,75, 7107 (2010); (r) D. Tan, V. Strukil, C. Mottillo and C.T. Friscic, Chem. Commun., 50, 5248 (2014); (s) K. Crossey, R.N. Cunningham, P. Redpath and M.E. Migaud, RSC $A d v$, 5, 58116 (2015); (t) I. IDokli and M. Gredicak, Eur. J. Org. Chem.,2727 (2015); (u) P.F.M, Oliveira, M. Baron, A. Chamayou, C. Andre-Barres, B. Guidetti and M. Baltas, RSC Adv., 4, 56736 (2014); (v) T.X. Metro, J. Bonnamour, T. Reidon, A. Duprez, J. Sarpoulet, J. Martinez and F. Lamaty, Chem. Eur. J,21. 12787 (2015); (w) T.K. Achar, S. Maiti and P. Mal, RSC Adv., 4, 12834 (2014).

28. K. Komatsu, Top. Curr. Chem., 254, 185 (2005), DOI: 10.1007/b100998

29. C.L. Raston and J.L. Scott, Green. Chem., 2, 49 (2000), DOI: 10.1039/A907688C

30. V.P. Balema, J.W. Wiench, M. Pruski and V.K. Pecharsky, J.Am.Chem.Soc., 124, 6244 (2002), DOI: $10.1021 / \mathrm{ja} 017908 \mathrm{p}$

31. G. Kaupp, M.R. Naimi-Jamal and J. Schmeyers, Tetrahedron, 59, 3753 (2003), DOI: 10.1016/S00404020(03)00554-4

32. J. Mackand M. Shumba, Green. Chem., 9, 328 (2007), DOI: 10.1039/B612983H

33. F. Schneider, T. Szuppa, A. Stolle, B. Ondruschka and H. Hopf, Green. Chem, 11, 1894 (2009), DOI: 10.1039/B915744C

34. T.L. Cook, J.A. Walker and J. Mack, Green. Chem., 15, 617(2013), DOI: 10.1039/C3GC36720G

35. E. Tullberg, D. Peters and T.J. Frejd, Organomet. Chem., 89, 3778 (2004).

36. K. Tanaka and F. Toda, Chem. Rev., 100, 1025 (2000), DOI: 10.1021/cr940089p

37. D. Cincic, I. Brekaloand B. Kaitner, Chem. Commun., 48, 11683(2012), DOI: 10.1039/C2CC36357G

38. O. Dolotko, J.W. Wiench, K.W. Dennis, V.K. Pecharsky and V.P. Balema, New. J. Chem., 34, 25 (2010), DOI: $10.1039 /$ B9NJ00588A

39. C.B. Aakeroy and A. S. Sinha, RSC Adv., 3, 8168(2013), DOI: 10.1039/C3RA40585K

40. I. Huskic, I. Halasz, T. Friscic and H. Vancik, Green. Chem, 14, 1597(2012), DOI: $10.1039 / \mathrm{C} 2 \mathrm{GC} 35410 \mathrm{~A}$

41. T.Z. Bhutia, P. Geethika, A. Malik, V. Kumar, B.G. Roy, M. Banerjee and A. Chatterjee, RSC Adv., 5, $99566(2015)$.

42. E. Colacino, P. Nun, M.F. Colacino, J. Martinez and F. Lamaty, Tetrahedron, 64, 5569(2008), DOI: 10.1016/j.tet.2008.03.091

43. Y. Serkan, H. Ozkan, N. Colak and Y. Yildirir, Molecules, 16(8), 6677(2011), DOI: 10.3390/molecules 16086677 
RASĀYAN J. Chem.

Vol. 12 | No. 4 |2183 - 2209| October - December | 2019

44. (a) T. Welton, Chem. Rev, 99, 2071(1999); (b) P. Wasserscheid and W. Keim, Angew. Chem. Int .Ed. Engl, 39, $3772 \quad$ (2000), DOI:10.1002/1521-3773(20001103)39:21<3772::AIDANIE3772>3.0.CO;2-5

45. R. Sheldon, Chem. Commun., 2399 (2001), DOI: 10.1039/B107270F

46. C.M. Gordon, Appl. Catal. A: Gen, 222,101 (2001).

47. J.S. Yadav, J.S.S. Reddy and R. Srinivas Rao, Tetrahedron, 59, 1599(2003), DOI: 10.1016/S00404020(03)00027-9

48. S. Kathiravan and R. Raghunathan, Syn. Commun., 43, 147(2013), DOI: $10.1080 / 00397911.2011 .59454$

49. S. Raju, A. Natarajan and A. Almamsour, Molecules, 20(1), 780(2015), DOI: $10.3390 /$ molecules 20010780

50. J. S. Yadav, B.V.S. Reddy and G.Baishya, J.Org.Chem., 68, 7098 (2003), DOI: 10.1021/jo0343351

51. O. Bortolini, D.A. Nino, L. Maiuolo, B. Russo, G. Sindona and A. Tocci, Tetrahedron Lett., 48, 7127 (2007), DOI: $10.1016 /$ j.tetlet.2007.07.219

52. (a) B. Chakraborty and G.P. Luitel, Tetrahedron Lett, 54, 765(2013), DOI: 10.1016/j.tetlet.2012.11.111; (b) B. Chakraborty, P.K. Sharma, M.S. Chettri, S. Kafley and A.R. Ghosh, Rasayan J. Chem., 2 (4), 946 (2009).

53. C.J. Li and T.H. Chang, Organic Reactions in Aqueous Media (Wiley: New York), 2007, 1997.

54. Y. Hayashi, Angew. Chem. Int. Ed (Eng), 45, 8103 (2006), DOI: 10.1002/anie.200603378

55. A.P. Brogan, T.J. Dickerson and K.D. Janda, Angew. Chem. Int. Ed(Engl), 45, 8100 (2006), DOI: 10.1002/anie.200601392

56. P.A. Grieco, Organic Synthesis in Water (Blackie Academic and Professional: London), 1998. U.M. Lindstrom, Organic Reactions in Water (Blackwell Publishing: Oxford), 2007, DOI: 10.1002/9780470988817.index

57. B.C. Ranu and S. Banerjee, Tetrahedron Lett., 48, 141(2007), DOI: 10.1016/j.tetlet.2006.10.142

58. S. Narayan, J. Muldoon, M.G. Finn, V.V. Fokin, H.C. Kolb and K.B. Sharpless, Angew. Chem. Int. Ed (Engl), 44, 3275 (2005), DOI: 10.1002/anie.200462883

59. J.E. Klijn and J.B.F.N. Engberts, Nature, 43, 7467 (2005).

60. R.N. Butler, W.J. Cunningham, A.G. Coyne and L.A. Burke, J. Am.Chem.Soc., 126, 11923 (2004), DOI: $10.1021 / \mathrm{ja} 040119 \mathrm{y}$

61. A. Chatterjee, K.A. Maiti and P.K. Bhattacharjee, Org. Lett., 5 (21), 3967 (2003), DOI: $10.1021 / \mathrm{ol} 035535 \mathrm{~m}$

62. D.G. Lez-Cruz, D. Tejedor, E.Q. Morales, F.G. Tellado and P.D. Armas, Chem. Commun., 2798 (2006), DOI: 10.1039/B606096J

63. R.K. Khangarot and K.P. Kaliappan, Eur. J. Org. Chem., 13, 7664 (2013), DOI: 10.1002/ejoc.201201599

64. C.S. McKay, D.C. Kennedy and J.P. Pezacki, Tetrahedron Lett., 50, 1893 (2009), DOI: 10.1016/j.tetlet.2009.02.035

65. A. Basak, K. Chandra, R. Pal and S.C. Ghosh, Synlett., 27, 1585 (2007).

66. Z. Chen, L. Lin, M. Wang, X. Liu and X. Feng, Chem. Eur. J., 19, 7561 (2013), DOI: 10.1002/chem.201204373

67. B. Chakraborty, P.K. Sharma and M.S.Chhetri, J. Heterocyclic Chem., 49, 1260 (2012), DOI: 10.1002/jhet.971

68. B. Chakraborty and G.P. Luitel, J. Heterocyclic Chem., 52, 726 (2015), DOI: 10.1002/jhet.2181

69. M. Pineiro and M.V.D. Teresa Pinho e Melo, Eur. J. Org. Chem., 5287 (2009), DOI: 10.1002/ejoc. 200900644

70. S. Kobayashi and K.A. Jørgensen, Cycloaddition Reactions in Organic Synthesis (Wiley-VCH Weinheim), 2002.

71. A. Diaz-Ortiz, A. Diez-Barra, A. De. La. Hoz, M. Moreno, M.J. Gomez-Escalonilla and A. Loupy, Heterocycles, 43, 1021 (1996).

72. F. Langa, A. Moreno, A. Draz-Ortis and L.D.A. Hoz, Eur. J. Org. Chem., 3659(2000), DOI: 10.1002/1099-0690(200011)2000:22\%3C3659::AID-EJOC3659\%3E3.0.CO;2-0 
RASĀYAN J. Chem. Vol. 12 | No. 4 |2183 - 2209| October - December | 2019

73. B.T. Nguyen, A. Martel, R. Dhal and G. Dujardin, J. Org. Chem., 73, 2621(2008), DOI: $10.1021 /$ jo702490w

74. B. Chakraborty, G.P. Luitel and M.S. Chhetri, J. Heterocyclic Chem., 54, 1611 (2017), DOI: $10.1002 /$ jhet. 2657

75. (a) B. Chakraborty, P.K. Sharma and S. Kafley, Green. Chem. Lett. Rev., 6 (2), 141(2013); (b) B. Chakraborty, N. Rai and M.S. Chettri, Rasayan J. Chem., 3 (1), 110 (2010).

76. V.K. Sony, J. Kim and E.J. Cho, Advanced Synthesis \& Catalysis, 2626 (2018), DOI: $10.1002 /$ adsc. 201800238.

77. R.A. Mekheime, K.M. Al-Zaydil, A. Al Shamaryl and K.U. Sadek, Green. Sustainable Chem, 1, 176 (2011).

[RJC-5480/2019] 